\title{
A Trace-Driven Analysis of Wireless Group Communication Mechanisms
}

\author{
Surendar Chandra ${ }^{1, *}$, Xuwen $\mathrm{Yu}^{2}$ \\ ${ }^{1}$ FX Palo Alto Laboratory, Palo Alto, CA 94304, USA, surendar@acm.org \\ ${ }^{2}$ VMware Inc., Palo Alto, CA 94304, USA, xyu@vmware.com
}

\section{Abstract}

Wireless access is increasingly ubiquitous while mobile devices that use them are resource rich. These trends allow wireless users to collaborate with each other. We investigate various group communication paradigms that underly collaboration applications. We synthesize durations when members collaborate using wireless device availability traces. Wireless users operate from a variety of locations. Hence, we analyzed the behavior of wireless users in universities, corporations, conference venues, and city-wide hotspots. We show that the availability durations are longer in corporations followed by university and then in hotspots. The number of simultaneously available wireless users is small in all the scenarios. The session lengths are becoming smaller while the durations between sessions are becoming larger. We observed user churn in all the scenarios. We show that synchronous mechanisms require less effort to maintain update synchronicity among the group members. However, distributed mechanisms require a large number of replicas in order to propagate updates among the users. For asynchronous mechanisms, we show that pull-based mechanisms naturally randomize the times when updates are propagated and thus achieve better performance than push based mechanisms. We develop an adaptive approach that customizes the update frequency using the last session duration and show that this mechanism exhibits good performance when the required update frequency intervals are large. We also show that for a given number of gossips, it is preferable to propagate updates to all available nodes rather than increasing the frequency while correspondingly reducing the number of nodes to propagate updates. We develop a middleware to illustrate the practicality of our approach.

Keywords: Wireless LAN, group communication mechanisms

Received on 07 October 2011; accepted on 16 February 2012; published on 03 August 2012

Copyright ( $) 2012$ Surendar Chandra and Xuwen Yu, licensed to ICST. This is an open access article distributed under the terms of the Creative Commons Attribution license (http://creativecommons.org/licenses/by/3.0/), which permits unlimited use, distribution and reproduction in any medium so long as the original work is properly cited.

doi:10.4108/mca.2012.07-09.e5

\section{Introduction}

Wireless laptops are replacing desktops as the primary computing platform for many users. In an article published in 2006, USA today [1] described the emergence of about 30 million nomadic users in the US. Gartner Dataquest predicted a yearly growth of $10 \%$ of these users. Recently, global sales of laptops exceeded that of desktops [2]. On our university campus, between 2005 and 2008, the number of active wireless devices rose from 5,027 to 13,051 while the number of wired student desktops dropped from 7,035 to 1,323. Similar trends were observed at other universities [3].

${ }^{*}$ Corresponding author. Email: surendar@acm.org
Contemporary laptops are resource rich while high speed cellular and wireless LAN networks are ubiquitous. BuddeComm [4] estimated a global deployment of over 200,000 wireless hotspots. These hardware and network trends allow laptops to support collaborations in which groups of users modify shared objects. Synchronous approaches support simultaneous modifications by the group members, while asynchronous approaches eventually propagate updates to all participants. Each of these approaches can be implemented using a centralized or a distributed approach.

The performance of these systems depends on the availability of the users' devices. Prior systems were designed when users predominantly used wired desktops. We investigate the behavior of prior systems for wireless users. A significant deviation in the 
behavior of wireless users from wired users could necessitate a redesign of existing collaboration systems.

Capturing such an update trace is difficult; collaboration applications may need to be instrumented in order to collect their usage statistics. A large scale deployment of modified applications is hard. Hence, we synthesize the duration when group members collaborate using information about when the users were online.

There are further complications in deciding when group members were online. Typical wireless LAN users exchange security credentials and associate with an access point (AP), acquire a DHCP address and other network resources and finally authenticate themselves with the system. Also, the collaboration system might be implemented as an application. Hence, users cannot be considered to be available until the user explicitly starts the necessary programs. Finally, these applications might allow the user to control when collaboration functionality was desirable. The trace collection mechanism should reflect the durations when the user is actually able to run and interested in the collaboration mechanisms.

Also, unlike desktop users [5], wireless users are mobile and work from many different locations $[1,6]$ : home, office and public venues. Ideally, we need to simultaneously monitor all wireless locations (schools, offices, hotspots etc.) in a geographic locale (city) in order to evaluate the system from the perspective of a mobile user who operates in these locales. However, such an analysis requires monitoring multiple administrative domains; currently unavailable to us and many researchers. An intrusive alternative is to install loggers in each monitored laptop.

In this paper, we analyzed the behavior of wireless users in different communities: university, corporate lab, conference venue and in a city-wide hotspot federation at different times and locations (spanning 2001 through 2008 at several locations in North America). We used publicly available traces from the CRAWDAD [7] archive. We also collected application level traces at a university. We compare the different traces from the perspective of group communication systems that might be utilized by these wireless users.

In all these traces, wireless LAN access was free. Hence, we assume that users exclusively used wireless LAN networks for collaboration (and did not also use a cellular network). Prior work $[3,8,9]$ observed significant local wireless traffic within an university. An article in the Economist [10] highlighted attempts to encourage more interaction among hotspot users and suggests that the nomadic users themselves are supportive of such efforts because of common purpose, lending further credence to our assumption.

We show that the durations that users were online decreased while the durations between sessions increased. The unavailable durations were longer for hotspots as compared to the university users. The system was dynamic with constant churn.

The observed availability behavior leads to poor performance regardless of the update propagation policy. Synchronous mechanisms encounter few group members who will simultaneously modify the shared contents. Distributed approaches require as many as 150 replicas in order to achieve acceptable update availability in the hotspot scenario. In general, asynchronous approaches are preferable for disconnected access. For small group sizes, server mediated approaches achieve better performance although for large groups, a distributed approach out-performed a server based approach. In general, a pull based approach leverages the randomness of group availability to achieve better performance than push based schemes. We showed that the immediate past session duration of an user was sufficient to predict the current session duration. However, systems that required frequent update propagations did not benefit from history based prediction as much as systems that propagated updates less frequently. Our approach is practical; we used the policies developed to propagate updates in our moderated collaboration system called flockfs $[11,12]$. We incorporate the lessons learnt into our Yenta middleware.

Section 2 analyzes the various availability traces. We investigate several synchronous and asynchronous group communications using these traces in Sections 3 and 4 , respectively. We describe related work in Section 5 with concluding remarks in Section 6.

\section{Wireless user availability analysis}

Analysis of group communications depends on knowing when users create updates. One way to collect this information is to instrument and widely deploy several applications that follow the different group communication paradigms. Instead, we synthesize the durations when users update contents by extrapolating from durations when users were available.

\subsection{Wireless traces analyzed}

We used publicly available access traces collected from a corporate research lab, a conference and a city-wide hotspot federation. We also collected application level wireless user availability traces from an university.

University campus (academia). Some of the large scale wireless user availability traces were collected at Dartmouth College. These traces include SNMP logs from Fall 2003 and Winter 2004 [13] as well as syslog traces from Sep. 2005 through Oct. 2006 [14]. The syslog traces record the association and disassociation events from each wireless device to an access point; the time between these records show the duration when a particular user was online. However, the syslog traces 
were recorded using unreliable UDP datagrams; many of the trace datagrams were apparently lost (confirmed through private email correspondence). Our analysis of the syslog entries from the Dartmouth Aruba routers [14] (after removing extraneous association messages we modified the syslog_parser script [15] to consider 'update station bssid to' records for AP associations) showed that there were 428,703 events with matching association and disassociation records. We also observed $5,966,261$ association events without the corresponding disassociation records (from the same access point) as well as 428,703 disassociation records without the corresponding association records. Hence, these syslog traces were not suitable for our study. Older SNMP logs were analyzed in $[3,16]$. We collected newer application level user availability information from our university.

On our campus, wireless networks are ubiquitous and deployed throughout the campus and the dormitories. The system used over 1,300 access points. We collected user availability traces using the Zeroconf protocol [17]. Users running Apple Mac OSX and Linux (with Avahi) reported when they became online (after successful association with an AP, acquiring an IP address and user authentication) and offline using the Zeroconf _workstation._tcp service. However, Zeroconf does not capture handover events across multiple access points within the same subnet. Users running the Apple iTunes client (on Microsoft Windows and Apple Mac OSX) report their availability using the _dacp._tcp service. Also, users who ran iTunes used the _daap._tcp service to advertise when the user explicitly consented to sharing their contents with other iTunes users. iTunes did not impose a tit-for-tat mechanism to fairly share contents; users are allowed to consume shared objects from other users while not sharing any objects on their own. Voida et al. [18] suggested that users share contents to express their individuality. Overall, the workstation reflects the user availability for asynchronous group communication systems, dacp highlights the potential availability for collaboration applications and daap shows the time that the user actually participated in iTunes like collaboration. iTunes only allows read-only sharing of objects and does not represent our target group collaboration systems. Regardless, these traces allow us to investigate the complexity of user availability.

Logistically, Zeroconf pushed the service availability information to the monitoring client using link local multicast. Since these packets are not routed, we required the service collection station to be co-located inside the monitored VLAN. We reconfigured the entire campus wireless network to use a single VLAN and placed our monitoring station on this wired distribution network of the wireless infrastructure. We used appropriate firewalls on the APs to prevent other types of network traffic from overwhelming the wireless networks. Without these filters, all broadcast packets will be sent to all the 1,300 APs. We collected the data for eleven days from Sep. 19, 2006 through Sep. 29, 2006 (our campus had 800 APs in 2006). We also collected the data from Dec. 3, 2007 through Aug. 25 , 2008. This period included the end of Fall '07 semester, winter break, Spring '08 semester, spring break and Summer '08 sessions. We place particular importance on the two weeks starting at 12/04/2007 (when users were likely to be collaboratively working on course projects, though not necessarily using any of the mechanisms explored in this paper) as well as on 60 days starting from 12/04/2007 (includes the busy end of semester season, calm winter break and the beginning of a new semester). Throughout this paper, we refer to these traces as workstation-2006, itunes-2006, share-2006, workstation-2008, itunes-2008 and share-2008. Note that the network administrators progressively removed groups of access points into their own (unmonitored) VLANs during the beginning of Spring '08 semester, spring break '08 and Summer '08, reducing the number of simultaneously available users. Our analysis of each segment showed that the user behavior was similar across all the time periods; albeit with fewer monitored users. Overall, the total number of users monitored in workstation-2006, itunes2006, share-2006, workstation-2008, itunes-2008 and share-2008 was 2,036, 4,893, 1,702, 4,063, 3,745 and 2,391 , respectively. The number of wireless devices was greater in 2008, although the number of iTunes users decreased from their 2006 levels. On the other hand, as compared to $34.78 \%$ of the iTunes users sharing their song collection in 2006 , over $63.85 \%$ of iTunes users shared their songs in 2008.

Corporation. Balazinska et al. [19] collected user availability traces at IBM Research from Jul. 22, 2002 through Aug. 17, 2002. Although these traces were collected before wireless networks were ubiquitous to the broader community, wireless laptops were already in widespread use among corporate users. These traces also remain the only publicly available trace of wireless users in a corporate setting. During the data collection period, IBM used 177 APs spread across three buildings (one of which was reportedly ten miles from the other buildings). For their traces, they issued SNMP probes ranging from every 5 (55\% of the traces) to up to 15 minute intervals. The probe duration affects the resolution of the user availability intervals. During the trace collection, they observed 1,366 wireless devices. They published their analysis of user and access point network load distribution in Mobisys 2003 [20]. We refer to these access traces as corporate-2002.

Conference. Conferences and other public congregations offer wireless access to an ephemeral collection of participants who likely share similar content interests. 
Many computer science conferences install their own wireless APs to provide wireless acces in the conference halls. Lately, the trend has been to utilize the wireless infrastructure available at the conference venue itself and provide free coverage to the participants throughout the conference venue: halls as well as the guest rooms. We have been unsuccessful in convincing these hotels to allow us to monitor the behavior of conference participants. One publicly available trace that contains user availability information was collected by Balachandran et al. [21] at the SIGCOMM 2001 conference (hosted at the UCSD campus). The traces were collected over two and a half days. Wireless coverage was provided using four access points in a single hall. The traces contain 195 distinct devices. The authors focused on the wireless traffic created by these users and presented their analysis in Sigmetrics 2002 [22]. We refer to these traces as sigcomm-2001.

Note that the wireless trace from the SIGCOMM 2008 conference [23] is not useful for our study due to data corruption, as much of the syslog traces except for 82 minutes on the last day were lost. Those traces did not reliably capture a disassociation event, making it hard to know the session durations. Additionally, the traces only captured users who voluntarily connected to a special IEEE 802.11a AP.

Hotspot. Finally, we analyzed the availability traces from a hotspot federation. ile Sans Fil (http://www. ilesansfil.org/welcome/) provides free coverage throughout Montreal, Canada using 206 hotspots; owners of public venues share their wireless networks that are then administered by ille Sans Fil. Lenczner et al. [24] released access traces for the three years from Aug. 28, 2004 through Aug. 28, 2007. These traces were collected when users authenticate themselves to any of the hotspots controlled by ile Sans Fil. The trace contained records of 69,689 wireless users. We place particular importance on the most recent two weeks (starting from 08/01/2007) for our analysis. We call these access traces hotspot-2007.

\subsection{Number of simultaneously available users}

First, we plot the number of simultaneously available users as a function of the time of day for the various traces in Figs. 1 and 2. When the number of simultaneously available users is high, synchronous mechanisms incur high overhead in propagating and maintaining updates from all the online members.

We note that users in all the traces exhibit a diurnal variation. Even corporate desktops which are stationary [5] exhibited some diurnal variations.

First we consider the behavior of users in academia. Fig. 1(d) and 1(a) plots the analysis of workstation-2008 and workstation-2006 traces, respectively. The first two weeks starting on 12/04/2007 of workstation-2008 were the weeks before final exams of the Fall 2007 semester. Many users were likely to be available working on their final projects. Hence, we pay particular attention to the first fifteen days of workstation-2008. Overall, we observed 2,036 new devices in workstation-2006, while in the first fifteen days of workstation-2008, we observed 2,729 new devices. In December 2007, we observed that the number of simultaneously available users reached a high of 410 users (15\% of users) with a diurnal low of two users late on a Sunday night. Note that wireless access is ubiquitous on our campus and many of our students live in the dormitories that were monitored in this study. By comparison, from workstation-2006 we detected as many as 775 users during the day times (38\% of the population). Even during late night hours, we observed over 200 simultaneously available users. Note that we do not know whether a wireless device was a laptop or a desktop (desktops are likely to be left online even when the user is not actively using them).

Similarly, we analyzed the application level availability of itunes-2008 and itunes-2006 in Figs. 1(b) and $1(\mathrm{e})$, respectively. Overall, we observed 2,617 users in the first fifteen days of itunes-2006 and 4,893 users in itunes-2008. The number of simultaneously available iTunes users reached $224(8.5 \%)$ in itunes-2008 and $989(20.1 \%)$ in itunes-2006. This drop in popularity is attributable to the drop in the session durations (Section 2.3). We also analyzed the scenario where users control the sharing duration for share-2008 and share2006 in Figs. 1(c) and 1(f), respectively. Of the 1,702 unique users from share-2006, we observed between 100 and 250 users $(6 \%-15 \%)$ were simultaneously available. For the first fifteen days of share-2008, we observed between 10 and 54 users (from a total of 2,391 users or $0.4 \%-2.3 \%$ ). The number of users who were willing to simultaneously share their iTunes collection dropped significantly between 2006 and 2008 even though the number of unique users sharing their contents had increased. User availability is complex and depends on the mechanism used for collecting the traces. Lower level mechanisms over-estimate the number of users that were willing to share their contents through iTunes. We expand on this observation in Section 2.3.

Next we analyzed the behavior of corporate-2002 traces in Fig. 2(a). We observed 1,366 unique devices. During the weekdays, the number of simultaneously available users reached as high as 529 (38.7\%). Even during late nights, we observed over 200 users who were available; but these were likely devices without any users actively using them. Over the weekend, the number of devices drops further to about 160; its likely that about 40 users (who left their wireless devices at work during the weekday nights) took their wireless devices home for the weekend. Similar analysis of sigcomm-2001 data in Fig. 2(b) showed that 119 of the 195 devices were available during the day time. Even 


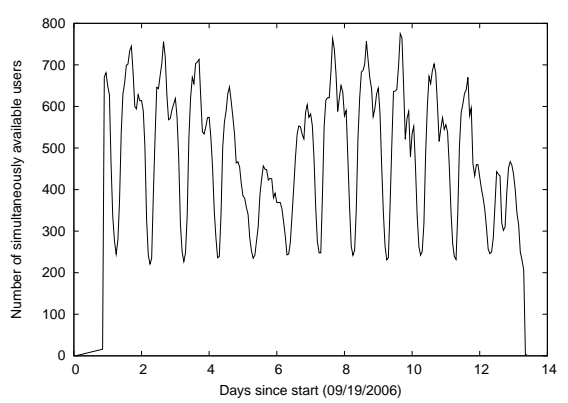

(a) workstation-2006

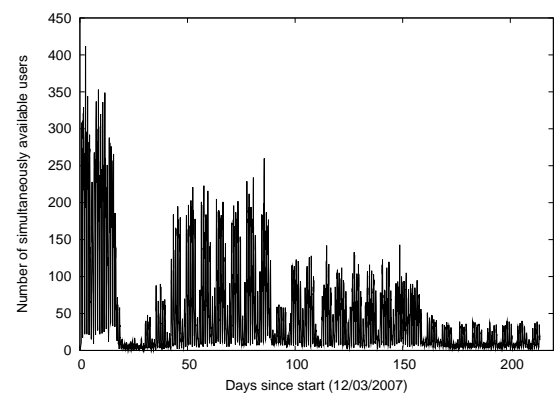

(d) workstation-2008

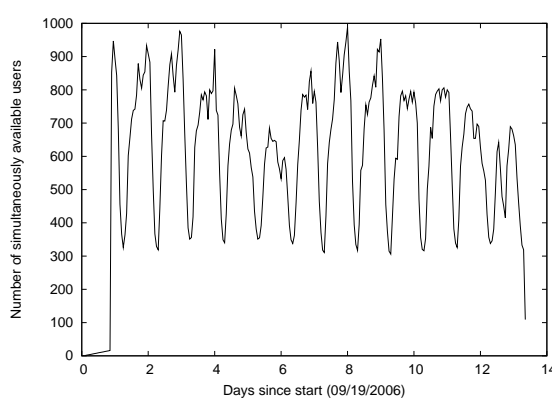

(b) itunes-2006

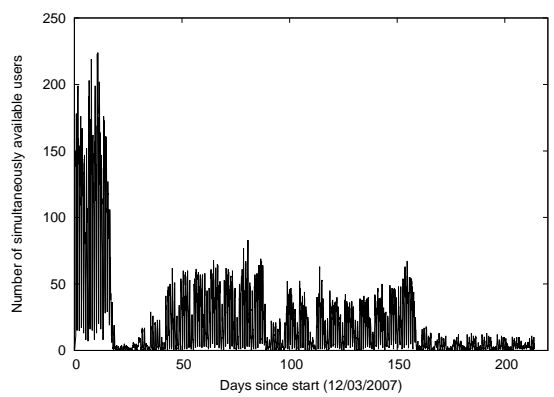

(e) itunes-2008

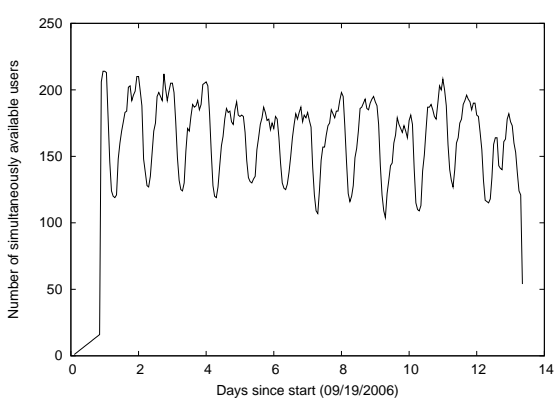

(c) share-2006

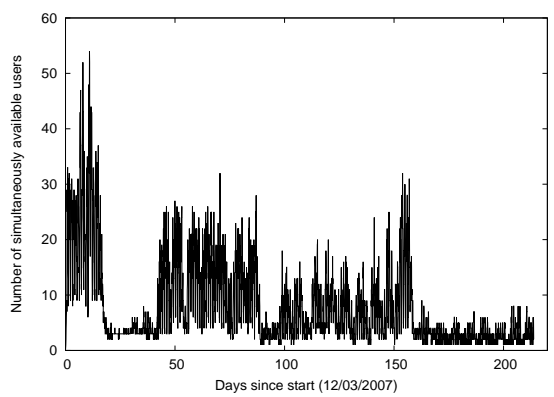

(f) share-2008

Figure 1. Number of simultaneously available campus users (academia)

during night times when the conference participants were likely to be unavailable, we still observed over 20 devices that were associated with the AP. Perhaps, these conference attendees were collaborating with colleagues in their home institutions located in a different time zone.

Hotspot-2007 traces span over three years with 70,000 users observed during the entire duration. In Fig. 2(c), we observed a steady increase in the number of simultaneously available users. During a two week duration starting in 8/1/2007 (Fig. 2(d)), we observed 2,725 unique devices; about 120 of them (4.4\%) were simultaneously available during the day.

\subsection{Session duration}

We analyzed the session characteristics and plot the amount of time that a user was available and the time between sessions for the various traces in Figs. 3 and 4 .

First, we plot the session behavior for the workstation2008 and workstation-2006 traces in Fig. 3(a). We also plot the values for the two weeks starting in $12 / 04 / 2007$. We note that the session durations in workstation-2008 traces were short; $50 \%$ of the sessions were under 20 minutes and $95 \%$ of the sessions were less than 75 minutes. Focusing our attention on the two weeks after $12 / 04 / 2007$, we note that $50 \%$ of the sessions were under 20 minutes and $95 \%$ of the sessions were less than 70 minutes. Sessions from workstation2006 were longer; $50 \%$ of the sessions were under one hour with $95 \%$ of the sessions were under 6.7 hours. The durations between sessions had increased from workstation-2006 to workstation-2008. For the workstation- 2008 trace, $50 \%$ of the durations between user sessions were less than 1.4 hours while $29 \%$ were longer than ten hours. Specifically focusing on the first two weeks in December 2007, these values were 1.2 hours for the median and $15 \%$ of the durations were longer than ten hours. On the other hand, workstation2006 showed that $50 \%$ of the durations between sessions was less than 47 minutes while $10.5 \%$ of the durations between sessions was larger than ten hours.

Even though the number of devices had increased (from 2,036 in workstation-2006 to 2,730 devices in the first two weeks of 2007), the session durations had decreased. Kotz et al. observed a median session length of 16.6 minutes in 2002 [16] and a reduction to under ten minutes in 2004 [3]. Chinchilla et al. [25] also observed that only $16.2 \%$ of sessions were less than one minute. Note that our trace collected using Zeroconf does not include IP resource acquisition and authentication durations included when analyzing AP SNMP logs. This change might be either because users were using less computing time or that the newer laptops offer a more reliable energy saving mode allowing the users to sleep (and hence become unavailable) longer. To understand this behavior, we analyzed the duration between successive arrivals of a particular user in order to understand whether the 


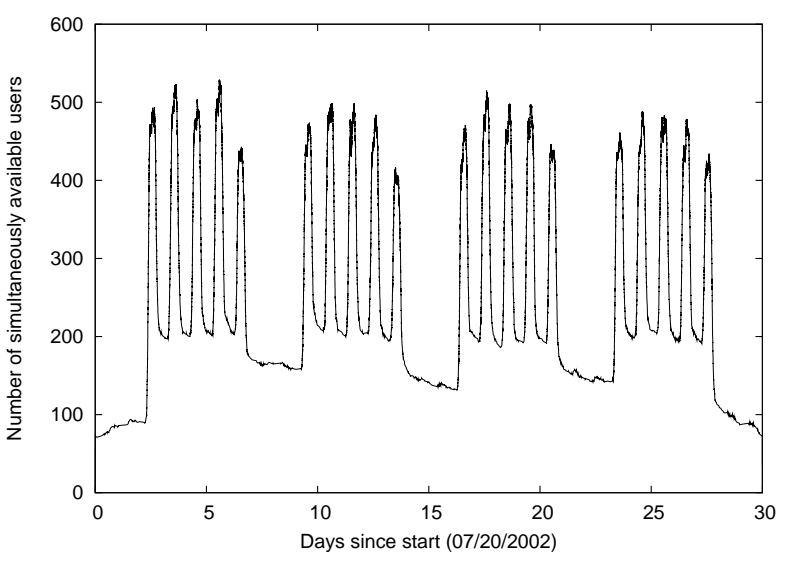

(a) corporate-2002

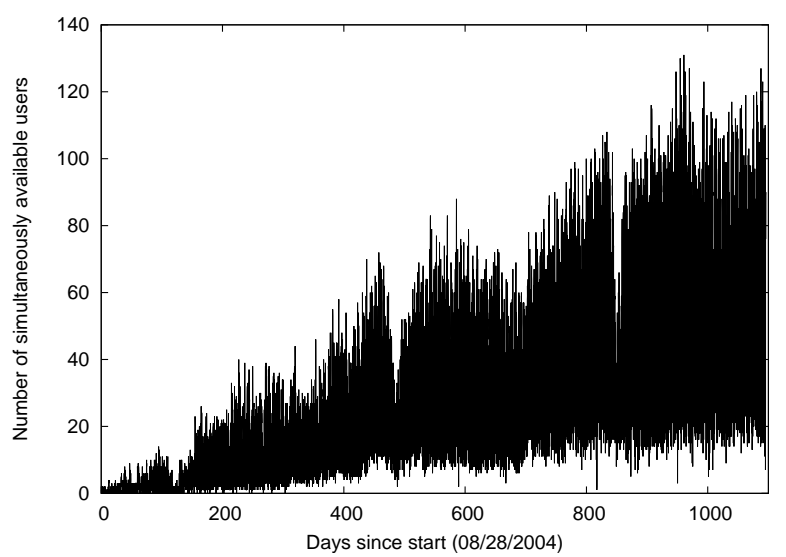

(c) hotspot-2007

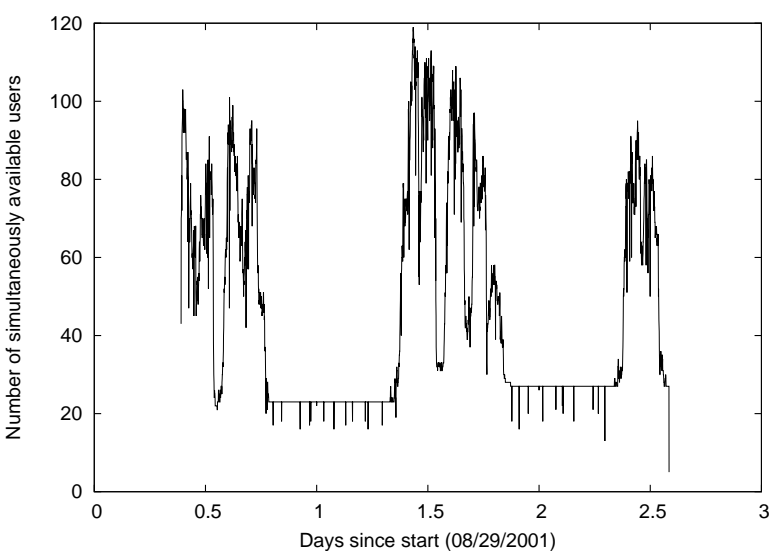

(b) sigcomm-2001

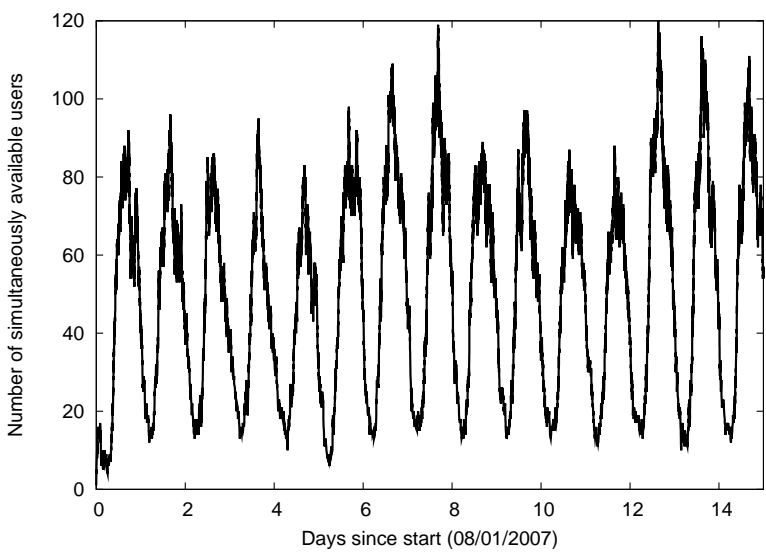

(d) hotspot-2007 (two weeks from 08/01/2007)

Figure 2. Number of simultaneously available users

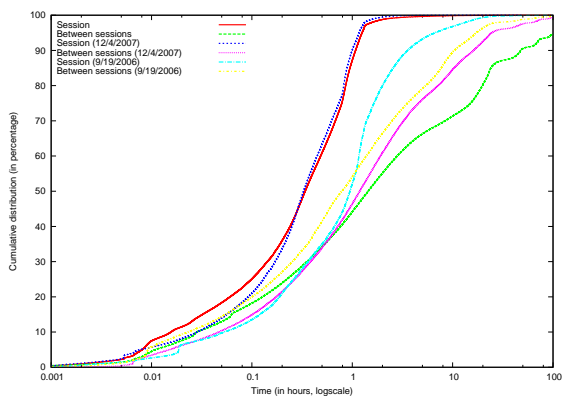

(a) workstation-2008 and workstation-2006

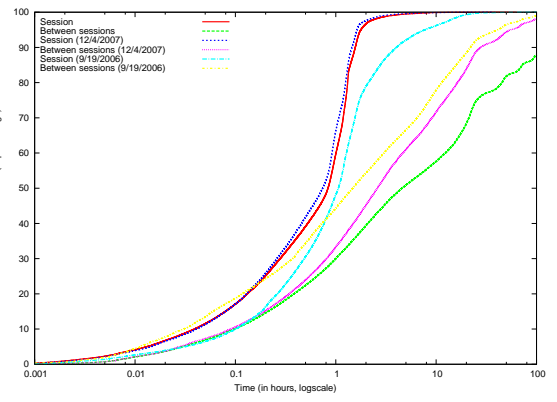

(b) itunes-2008 and itunes-2006

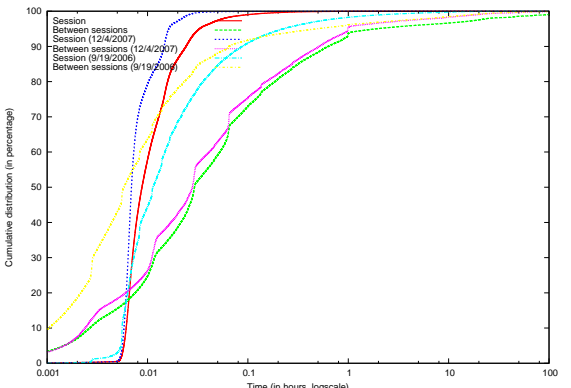

(c) share-2008 and share-2006

Figure 3. Online availability behavior of campus users (academia)

shortening session durations equaled the increase in duration between sessions. For the first two weeks in December 2007, median values were 1.78 hours while $75 \%$ of users online every 5.5 hours. In 2006, the median values were 2.52 hours with $75 \%$ of users online every 6.9 hours. The users whom came online often remained online for shorter durations. We will later show the performance reduction caused by this session duration change.

Next, we analyzed the session behavior for the itunes2008 and itunes-2006 traces (Fig. 3(b)). Note that prior trace collection efforts had not measured application level session availability durations. We note that $50 \%$ of the iTunes sessions from itunes-2008 were less than 48 


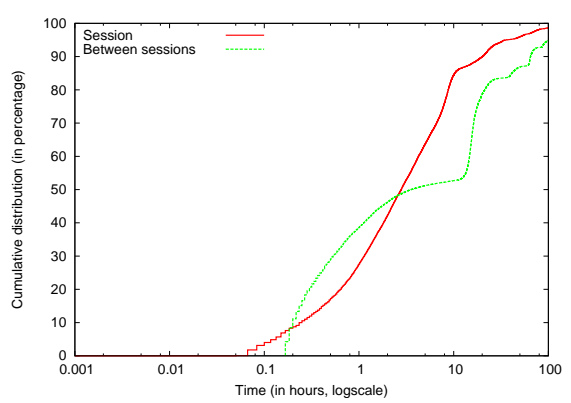

(a) corporate- 2002

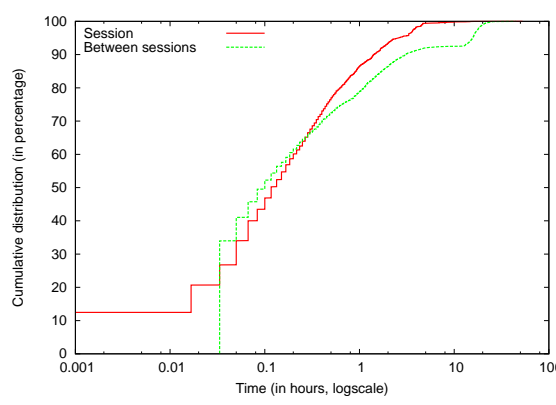

(b) sigcomm-2001

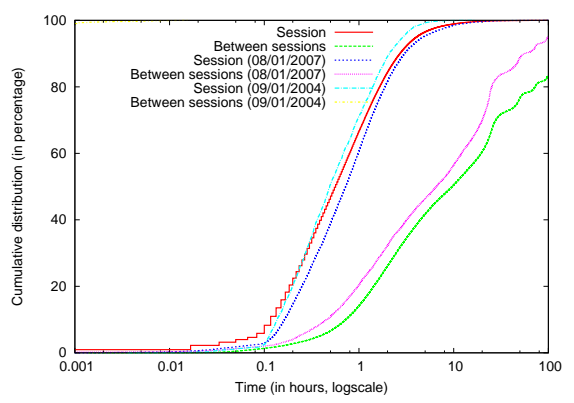

(c) hotspot-2007

Figure 4. Online availability behavior

minutes, while $95 \%$ of the sessions were less than 1.75 hours. Specifically focusing on the two periods since $12 / 04 / 2007$, we note that $50 \%$ of the sessions were less than 45 minutes, while $95 \%$ of the sessions were less than 100 minutes. However, for the itunes-2006 traces we note that $50 \%$ of the sessions were larger than one hour with $95 \%$ of the sessions being larger than 7.7 hours. The duration between sessions was even larger; itunes-2008 showed that $50 \%$ of the duration between iTunes sessions was less than 4.5 hours with $95 \%$ of the durations over ten hours. However, analysis of the itunes-2006 traces showed that 50\% of the duration between iTunes sessions lasted for less than 1.5 hours with $22 \%$ over ten hours. These trends are similar to the workstation traces.

We plot the session characteristics for the share-2008 and share-2006 traces in Fig. 3(c). Session durations when users shared their iTunes collection were small. For the share-2008 traces, over $96 \%$ of the sessions were under 0.01 hours while $50 \%$ of the sessions were under 0.01 hours for the share-2006 traces. Over $90 \%$ of the durations between sessions for the share-2008 traces were under an hour while the corresponding measures for the share-2006 traces were under 0.1 hours. The users exert control over when they shared their songs.

For the corporate-2002 traces (Fig. 4(a)), we note that $50 \%$ of the sessions were longer than 2.8 hours with $95 \%$ of the sessions less than 36 hours. Analyzing the duration between sessions, we note that $50 \%$ of them were over 3.5 hours with $48 \%$ over 10 hours. Similarly, for the sigcomm-2001 traces (Fig. 4(b)), we note that most sessions and duration between sessions were small; $86 \%$ of the sessions were less than one hour long with only $22 \%$ of the duration between sessions longer than one hour. It is likely that conference attendees were paying attention to the conference and were using wireless networks for brief intervals. On the other hand, analyzing the hotspot-2007 traces (Fig. 4(c)), we note that $50 \%$ of the sessions were smaller than 35 minutes with $95 \%$ of the sessions less than four hours. Similarly, analyzing the Verizon hot spot network in
Manhattan, Blinn et al. [26] observed that $45.74 \%$ of the sessions lasted more than one hour. The duration between sessions for our hotspot traces was over 9.6 hours for $50 \%$ of the cases with $49 \%$ of the duration between sessions longer than ten hours.

We also analyzed the long term daily average session duration for workstation-2008, itunes-2008, share-2008 and hotspot-2007 and plot the results in Fig. 5. For workstation-2008 (Fig. 5(a)), we note that the session lengths are longer during breaks (weekend, winter, spring and summer breaks) than when classes were in session. At the application level, these variations were less pronounced for itunes-2008 (Fig. 5(b)) where we saw a slight trend towards longer session durations. The session lengths for share-2008 remained small during the semester and increased during the breaks. For the hotspot-2007 traces (Fig. 5(d)), we note a slight increase in the session duration with less variability, especially when compared to the first six months.

By contrast, the Farsite study [5] used ping messages on a corporate desktop environment and reported that most machines were always available with unavailable intervals occurring during weekends. They reported that the median number of machines were available for more than $95 \%$ of the time. The Farsite study observed that the unavailable durations occurred during late nights and on the weekends.

Variations based on the capture mechanism. The session analysis (Section 2.3) showed the differences in session lengths depend on whether the data was captured at the workstation or by a sharing application (e.g., iTunes). In order to understand the availability, we breakdown the session analysis for the 2006 traces into different time ranges and report our observations in Fig. 6. For these experiments, we divided the day into three time ranges: $12 \mathrm{AM}$ to $9 \mathrm{AM}$ (late night to early morning), $9 \mathrm{AM}$ to 5 PM (typical corporate work time) and 5 PM to 12 PM (evening). We analyzed the session durations and the period of unavailability between sessions for the three traces studied: workstation-2006, itunes-2006 and 


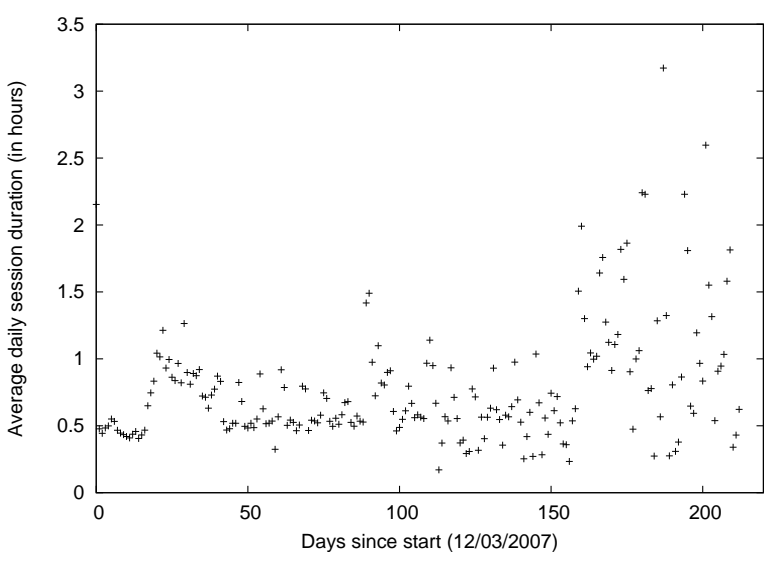

(a) workstation-2008

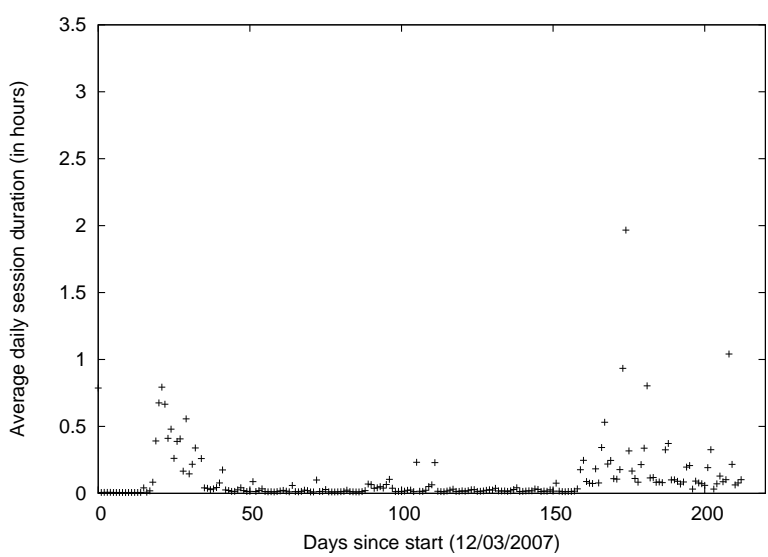

(c) share-2008

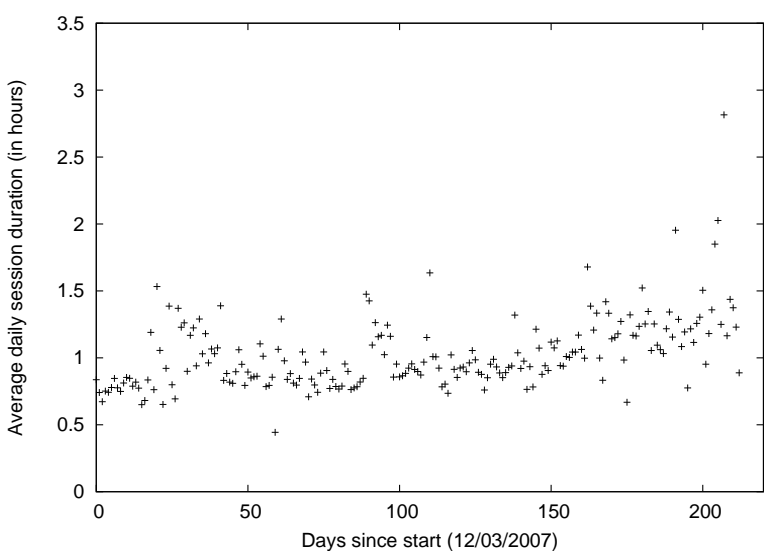

(b) itunes-2008

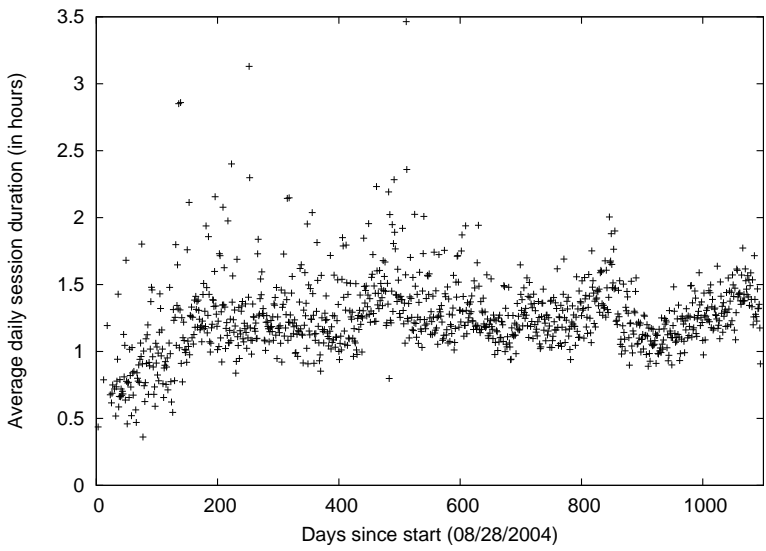

(d) hotspot-2007

Figure 5. Evolution of session duration

share-2006 and plotted the cumulative distributions. For sessions that spanned multiple time ranges, we attributed the durations to the starting time range. Most sessions were short and so this choice did not have an significant impact.

Analyzing the session durations for the workstation2006 (Fig. 6(a)), we noted that the system behavior was similar for the duration of 9 AM - 5 PM and 5 PM - 12 PM. The session durations were shorter during late night hours. For example, the median durations were about twenty minutes as compared to an hour for the rest of the day. $20 \%$ of the day time and evening users were available for more than 1.5 hours. Also, an analysis of unavailable durations from Fig. 6(d) showed that late night users were either available for two hours or available for long durations; $70 \%$ were available for less than two hours while the remaining $30 \%$ were available for over 6 hours. This behavior was consistent with users who either turn off their laptops before they go to sleep or leave them $\mathrm{ON}$ throughout the night. We observed no such behavior during the other durations. Over $90 \%$ of the evening users returned after less than three hours, while $10 \%$ of the morning users were unavailable for over eighteen hours. One likely explanation was that the work day users included staff who returned the following day while evening users included dormitory students who became available after dinner.

itunes-2006 traces were similar to the workstation2006 traces (Figs. 6(b) and 6(e)). 60\% of the users were unavailable for less than an hour or for the entire eight hours. The session duration did not show significant difference across the various time ranges. However, share-2006 traces exhibited oscillatory behavior where users became available and unavailable for short periods of time (both for the session durations (Fig. 6(c)) as well for the unavailable durations (Fig. 6(f))).

Our analysis shows the complexity of user availability. Further work is required to capture availability traces from deployed applications. We are collecting such traces for our collaborative system [11, 12] designed for wireless users. 


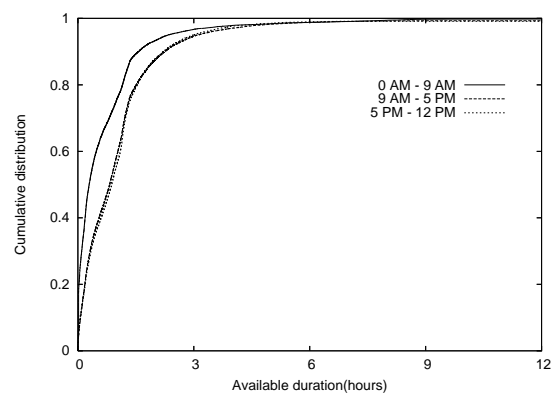

(a) Session: workstation-2006

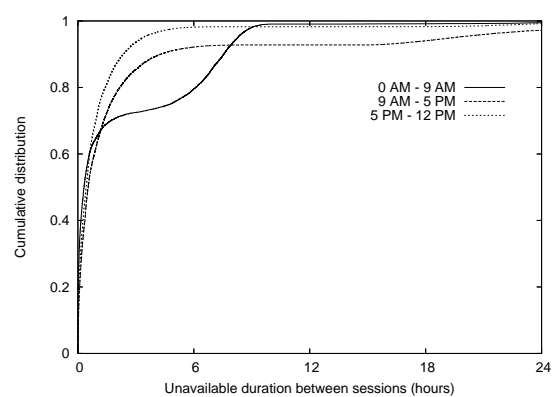

(d) Unavailable duration - workstation-2006

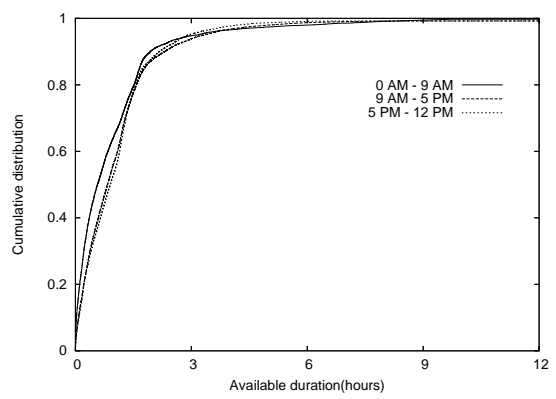

(b) Session: itunes-2006

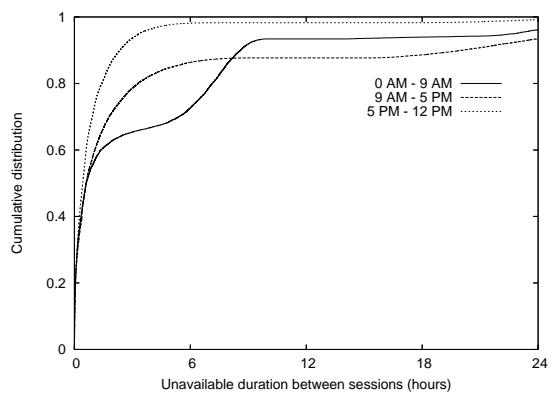

(e) Unavailable duration - itunes-2006

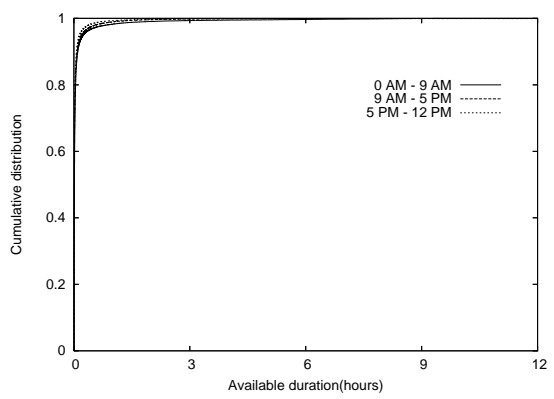

(c) Session: share-2006

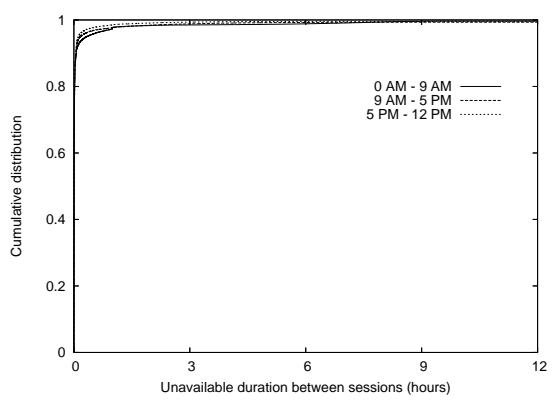

(f) Unavailable duration - share-2006

Figure 6. Session durations in different time ranges

\subsection{User churn behavior}

Next, we investigate the introduction and attrition of nodes for the various traces in Fig. 8. A new node will need to receive all the updates from prior users. In the academic scenario, we expect new users to arrive at the beginning of a semester while generally remaining stable throughout the semester. Analyzing the workstation- 2008 traces in Fig. $7(\mathrm{~d})$, we note that over 2,750 users (of the total 4,000 users) were seen within the first few weeks. However, after the winter break (about 50 days since the start of the trace), new users were steadily added even though the overall number of monitored APs was continuously reduced. On the other hand, about 500 users were never seen after the Fall 2007 semester (first few weeks). During the Spring 2008 semester, users constantly left the system until about 150 days into the trace. Note that APs were removed from our monitoring abruptly in the middle of the spring break (and not gradually as indicated by Fig. 7(d)). Similar results were observed for analyzing the itunes-2008 trace (Fig. 7(e)) and the corporate-2002 traces (Fig. 8(a)) where about $50 \%$ of the users appeared at the beginning of the traces (delayed because some users were likely offline during the start of the trace collection). However, the rest of the users continued to trickle in during the remainder of the trace duration (with a two day jump, likely because of the weekend). We even observed this effect in the sigcomm2001 traces (Fig. 8(b)) where the short duration of the traces made such a behavior seemingly unlikely; we observed that about 35 users joined the system after the first day. Finally, we observed that new users were constantly entering the system and existing users were similarly leaving the system by analyzing the hotspot2007 traces (Fig. 8(c)). This is likely typical in hotspots.

\subsection{Summary of results}

We analyzed the availability traces from academia, a corporate lab, a conference and a hotspot federation. An analysis of users in academia showed that session durations are becoming shorter and period between sessions are getting larger; both at the machine level as well as for the iTunes sessions. Students in academia are inherently mobile; roaming between their dormitory, classes, cafeterias etc. Anecdotally, newer wireless devices are more reliable for power saving sleep cycles; perhaps users are only using their wireless devices when they need to. In the corporate setting traces from 2002, the session durations were longer. In a corporate setting, most users have an office which will allow them to remain online for prolonged durations. The users in the hotspot remain online for short periods and then stay offline for long durations. These observations manifest themselves in fewer users (e.g., a smaller percentage of users) being simultaneously available in the recent traces from academia and in the wireless hotspots. In the next sections, we 


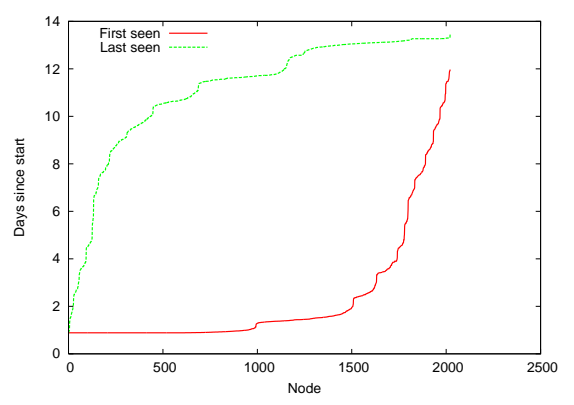

(a) workstation-2006

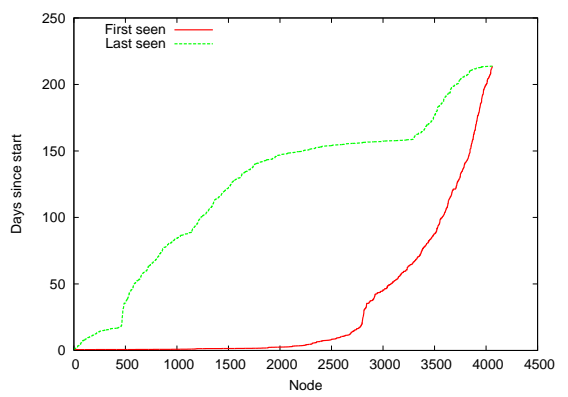

(d) workstation-2008

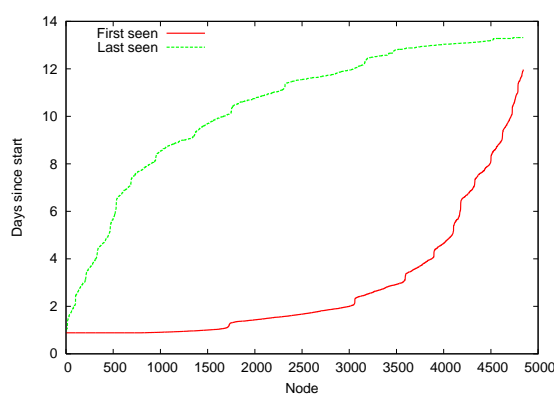

(b) itunes-2006

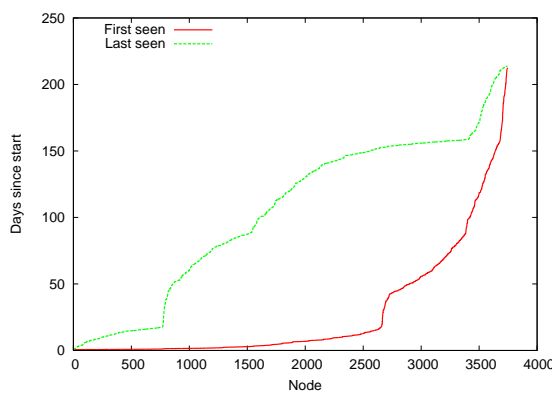

(e) itunes-2008

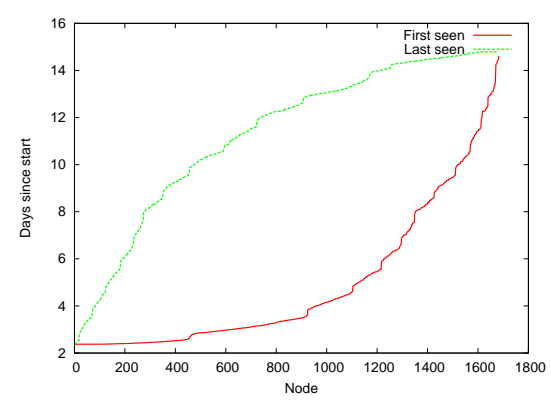

(c) share-2006

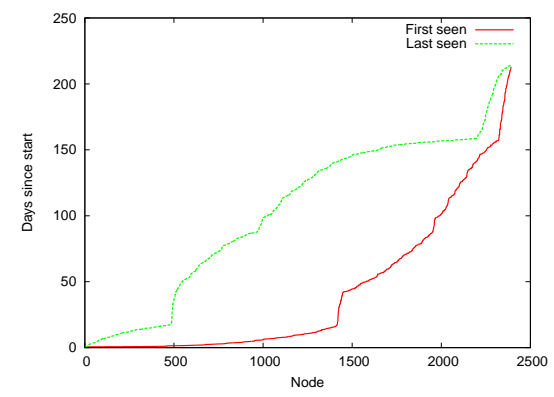

(f) share-2008

Figure 7. User churn behavior (academia)

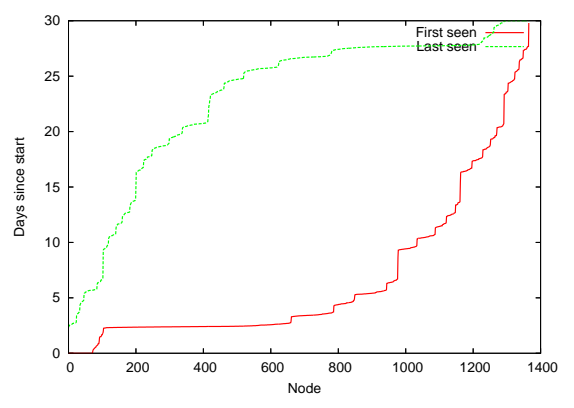

(a) corporate- 2002

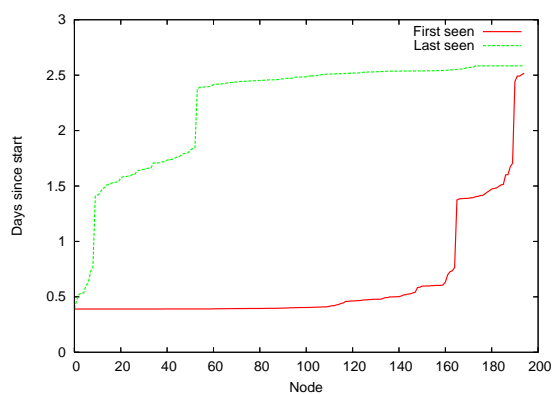

(b) sigcomm-2001

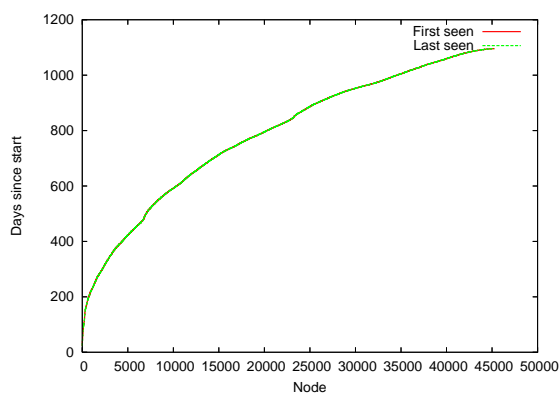

(c) hotspot-2007 (both the values overlap)

Figure 8. User churn behavior

investigate the implications of these observations for group communication mechanisms.

Next, we investigate various group communications mechanisms. Collaboration applications use group communication mechanisms to implement their collaboration functionality. We consider applications which allow any group member to modify shared contents. The system attempts to reflect each update to all the other group members. The group communication mechanism itself does not guarantee any message delivery order; although group collaboration applications may impose application specific ordering constraints. Also, we investigate the average behavior among a random group of wireless users. This random group should represent a lower bound; actual collaborating group members will exhibit higher correlated availability. We choose representative traces for ease of illustration.

\section{Synchronous mechanism}

Synchronous mechanisms support concurrent modifications of shared objects by all group members. Conflicting updates by different group members are addressed by either exclusively locking the shared object or using optimistic mechanisms [27]. Without conflicting updates, centralized approaches spend little effort in maintaining consistency. However, distributed approaches still need to propagate updates from the group member who updated the contents to other group members. Some examples of centralized synchronous systems include storage systems 


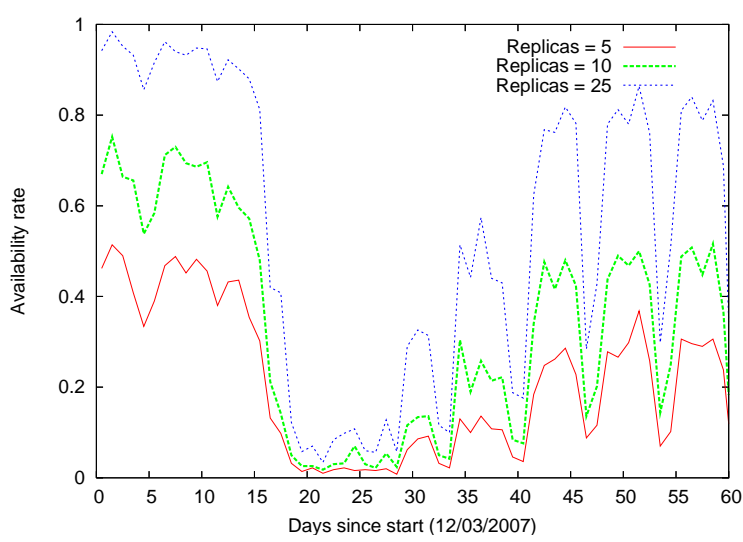

(a) workstation-2008 (max values in a day)

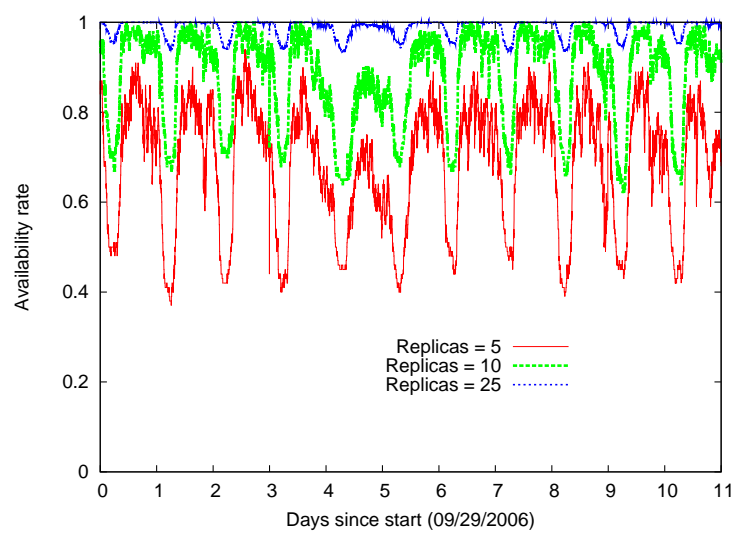

(c) workstation-2006

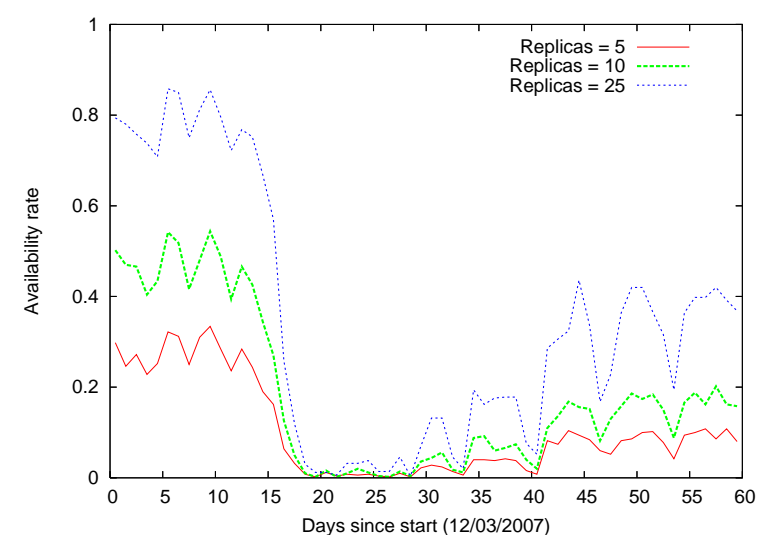

(b) itunes-2008 (max values in a day)

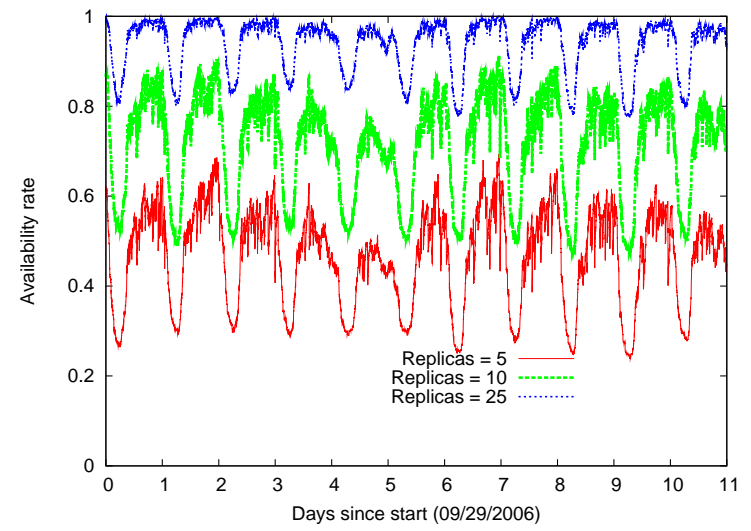

(d) itunes-2006

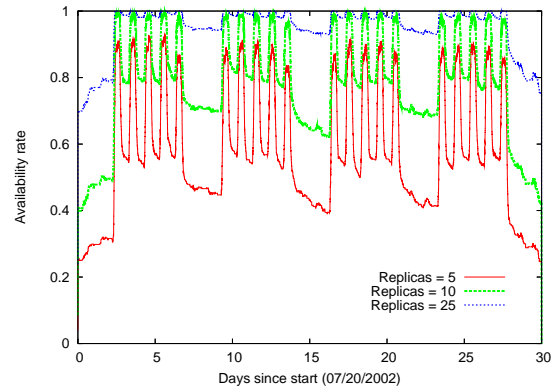

(e) corporate- 2002

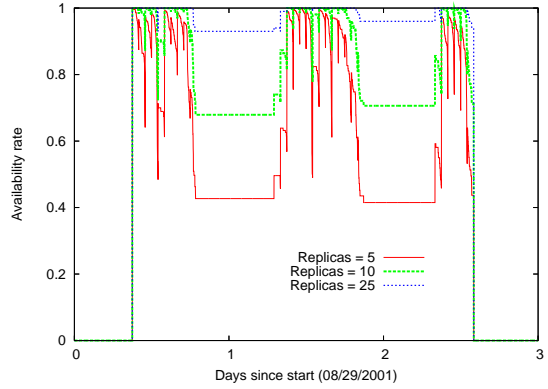

(f) sigcomm-2001

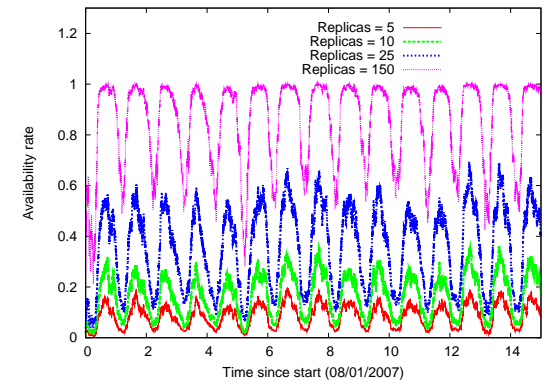

(g) hotspot-2007

Figure 9. Improving availability of local updates for other group members by replicating objects among wireless devices

such as AFS [28] and NFS [29] as well as applications such as MoonEdit (moonedit.com) and GoogleDocs (docs.google.com). Applications such as UNA (n-brain.net) and SubEthaEdit (codingmonkeys.de/ subethaedit) use a distributed approach.

In Section 2.2, we showed that few users were simultaneously available. For the more recent wireless users in academia, only $15 \%$ of the users were simultaneously available during the daytimes. These figures were even lower for the hotspot users where only $4 \%$ of the users were simultaneously available.
On the other hand, $38.7 \%$ of corporate users were simultaneously available during the daytimes. These figures were far lower during night times. By comparison, Farsite [5] observed high availability among wired corporate desktops. On average, the number of conflicting updates among wireless group members who were simultaneously available is likely to be small. Conflict resolution techniques designed for wired users should be adequate for wireless users.

Since not all group members are simultaneously available, the challenge is to propagate each update 
to other group members as they become available. One way to increase availability is to use a wired server for storage. Another alternative is to use the wireless devices themselves. Since contemporary wireless devices are resource rich, we evaluate the amount of replication required to achieve good availability of specific contents. Such evaluations had been performed using desktop storage [30, 31]. Using corporate desktops, Farsite [32] achieved four nines of availability using just three replicas.

We randomly select five, ten and twenty five replicas. We assume that these nodes were simultaneously available with the originating node and so the updates were directly replicated in all these nodes. Using asynchronous mechanisms [33] to propagate updates to these replicas can itself introduce long delays. We calculate the availability of this particular content to others throughout our trace interval. In effect, this is the best case scenario where the group member that requires the update can be any node from our trace other than the replica nodes. Note that we only need one of these replicas to be online in order to make the contents available. We repeated each experiment 1,000 times and plot the average availability by the time of the day for the various traces in Fig. 9.

First we analyzed the system in academia. For the workstation-2008 and itunes-2008 traces, we improve readability by plotting the maximum availability values in a particular day. In general, the availability follows a diurnal as well as a seasonal pattern (end of semester, winter break, beginning of new semester). Analyzing the workstation-2008 traces (Fig. 9(a)), note that five replicas only achieved about $50 \%$ availability during December 2007. The availability reduced to almost $0 \%$ during the winter break before recovering to about $20 \%-30 \%$ in the Spring 2008 semester. Increasing the replication rates to ten replicas improved the availability in December 2007 to $70 \%$ while 25 replicas are required to achieve $100 \%$ availability (albeit briefly). Comparing these results with the workstation-2006 traces (Fig. 9(c)), we observed that five replicas achieved up to $90 \%$ availability while 25 replicas achieved $100 \%$ availability consistently during daytimes. Reduced session durations is worsening the prospect for distributed update propagation.

An analysis of itunes-2008 showed that the availability was worse for iTunes users. Fig. 9(b) shows that itunes-2008 users only achieve about $40 \%$ availability for using five replicas in December 2007. Even when we increase this amount to 25 replicas, the availability only reaches $90 \%$. On the other hand, by the beginning of Spring 2008, even with 25 replicas, the maximum availability only reaches around $40 \%$. By contrast, an analysis of itunes-2006 in Fig. 9(d), shows that 25 replicas was enough to provide $100 \%$ availability during the daytimes. Even five replicas achieved about $60 \%$ availability for a targeted contents during the day times.

Next we analyzed the behavior in a corporate setting using corporate-2002. We note that (Fig. 9(e)) five replicas provide about $90 \%$ availability during the weekday business hours. During the evenings, we achieve availability of about $60 \%$ which reduces to about $45 \%$ by the weekend. Replications of about 25 copies achieve about 95\% availability even during the weekend. Note that Farsite [32] achieved excellent availability using only three desktop replicas.

The sigcomm-2001 traces (Fig. 9(f)) show that even five replicas achieved good availability during the conference durations. However, 25 replicas are required to achieve good availability during the night hours when the conference was not in session. Finally, we analyzed the behavior for the hotspot2007 traces. In Fig. 9(g), we note that five replicas only achieve about $10 \%$ availability. Even 25 replicas only achieves about $60 \%$ availability during daytimes. We required about 150 replicas to achieve $100 \%$ availability during the weekdays. Distributed update propagation is not feasible among wireless devices in a hotspot; the hotspot provider should provide the storage infrastructure necessary to make shared contents available to other group members.

To summarize, the number of simultaneously available users and the duration that each user was available is decreasing. Unlike wired scenarios, this trend reduces the effort required to maintain consistency among updates. However, this exacerbates the problem of distributing updates from one group member to all others. Distributed approaches require a large number of replicas to forward updates to other members. Even though laptops are resource rich and capable of providing service to others, the replica requirement makes a distributed approach impractical, especially in hotspots where we require over 150 replicas. Hotspots are also unlikely to provide the necessary storage servers. The performance achieved in a university and corporate scenarios were tolerable, especially during durations when all the users were active (e.g., daytimes). Next, we investigate asynchronous mechanisms.

\section{Asynchronous mechanism}

Asynchronous mechanisms modify local copies of shared contents. Updates are eventually propagated to other group members. Because of the update propagation delay, collaboration applications explicitly reconcile updates from other group members. Propagation and reconciliation can be mediated by servers (e.g., Coda [34], Apple iDisk (apple.com/mobileme) and Windows SkyDrive (skydrive.live.com)) or through distributed mechanisms (e.g., Ficus [35], Bayou [36] and Windows Live Sync (sync. live.com)). 
Update propagation can be implemented as a daemon process that starts as soon as the users authenticate themselves with the system. Since workstation-2008 traces capture this duration, we illustrate the system performance using this trace.

\subsection{Propagation policies}

A distributed approach propagates the updates to simultaneously online group members, likely delayed from when the update was created. Each update is subsequently propagated to other nodes through successive gossips in order to eventually reach all group members. Some of these gossip sessions are unnecessary because the corresponding pair of nodes already have all the updates available. Frequent propagation attempts will improve system performance while incurring a high number of unnecessary gossips. For example, Bayou [36] used version vectors to identify updates that needed to be propagated during a pairwise anti-entropy session. We investigate the impact of various policy parameters for update propagation.

Updates can either be pushed by the originating node or pulled by other nodes. We refer to these policies as P2P-push and P2P-pull, respectively. Similarly, a server mediated approach uses a (always online) server. This mediation can either be initiated by the node or the server. In server initiated mechanisms, the server periodically pulls updates from nodes that are online and then pushes them to other online nodes. We refer to this policy as Svr-ServInit. On the other hand, in a node initiated policy, the node that created updates periodically pushes the updates to the server while also retrieving updates from other nodes from the server. We refer to this policy as Svr-NodeInit. When they come online, nodes always initiate a push or pull operation.

The time to perform each push and push operation depends on the size of the update as well as on the available network bandwidth. Given the bandwidth availability on wireless LANs, we do not model this duration (similar assumptions as Demers et al. [37] and Birman et al. [38]). We assume that nodes go offline without explicitly pushing its updates to other nodes.

Depending on the group size, we also need to choose several other propagation parameters. During the initial phases, few nodes contain the updates and hence the system has to aggressively propagate the updates before the nodes that contain them go offline. This is particularly important while the update was only available in the node that created the update.

\subsection{Performance metrics}

Unlike metrics used by Vahdat et al. [39] that measured the time required to propagate a single update from a random node to every other group member, we use metrics that capture a dynamic system where

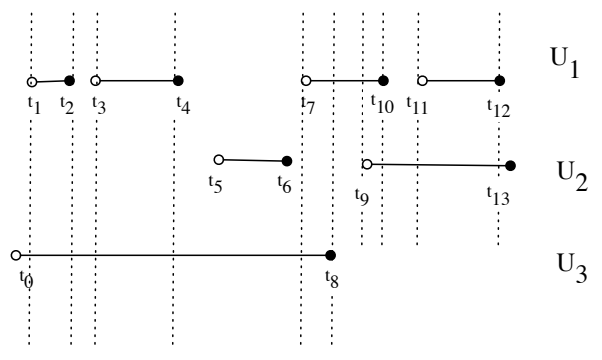

Figure 10. illustration of availability behavior of three users

new updates are continuously created by each group member. Consider a group of three nodes $\left(U_{1}, U_{2}\right.$ and $U_{3}$ ) and their availability durations as illustrated in Fig. 10. $U_{1}$ is available from $t_{1} \ldots t_{2}, t_{3} \ldots t_{4}, t_{7} \ldots t_{10}$ and $t_{11} \ldots t_{12} ; U_{2}$ is available from $t_{5} \ldots t_{6}$ and $t_{9} \ldots t_{13}$ and $U_{3}$ is available from $t_{0} \ldots t_{8}$. Say at $t_{8}$, our metrics quantify the amount of updates from $U_{1}, U_{2}$ and $U_{3}$ that are unavailable in the other nodes. Rather than choosing arbitrary values for update creation rates, we assume that nodes create updates at a constant rate. Hence, the amount of time that a node was available measures the updates created by the node. Consider a node that was missing updates created by another node that was online for two hours. A target application might create updates at the rate of one update per hour. For this application, this system performance would translate to missing two updates.

We quantify the number of updates using a lag metric and the network overhead by the number of gossips.

- lag metric: We measure the average number of updates that are unavailable at a node using lagAmount. Consider the illustration in Fig. 10 (ignoring $U_{3}$ for now). At $t_{5}, U_{2}$ does not have updates $\left(t_{1} \ldots t_{2}\right)$ and $\left(t_{3} \ldots t_{4}\right)$ from $U_{1}$; the lagAmount at $U_{2}$ is $\left(t_{2}-t_{1}\right)+\left(t_{4}-t_{3}\right)$. At $t_{6}$, the lagAmount of $U_{1}$ is $t_{6}-t_{5}$ and for $U_{2}$ is $\left(t_{2}-t_{1}\right)+\left(t_{4}-t_{3}\right)$ for an average lagAmount of $\frac{\left(t_{2}-t_{1}\right)+\left(t_{4}-t_{3}\right)+\left(t_{6}-t_{5}\right)}{2}$. The lag values depend on the propagation policies (further explored in Section 4.4). For example, $U_{3}$ could help in ferrying updates between $U_{1}$ and $U_{2}$.

- Gossips: numGossips measures the number of pairwise anti-entropy operations. If a particular gossip only considered updates from the corresponding node (without propagating updates from other peers), we consider these to be wasted gossips (numWGossips). Note that these metrics do not account for the size of updates (for example, measured in kilobytes) that were actually propagated during a successful gossip operation; this assumption was also used by prior work $[37,38]$. 


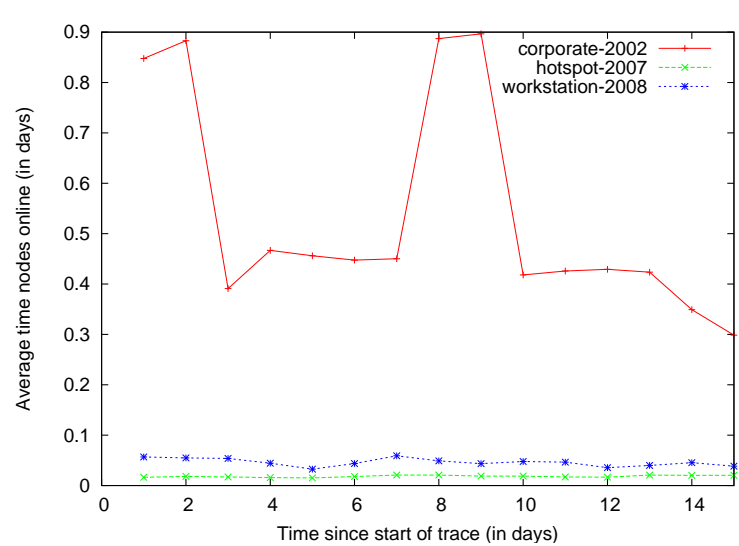

(a) Average time nodes online

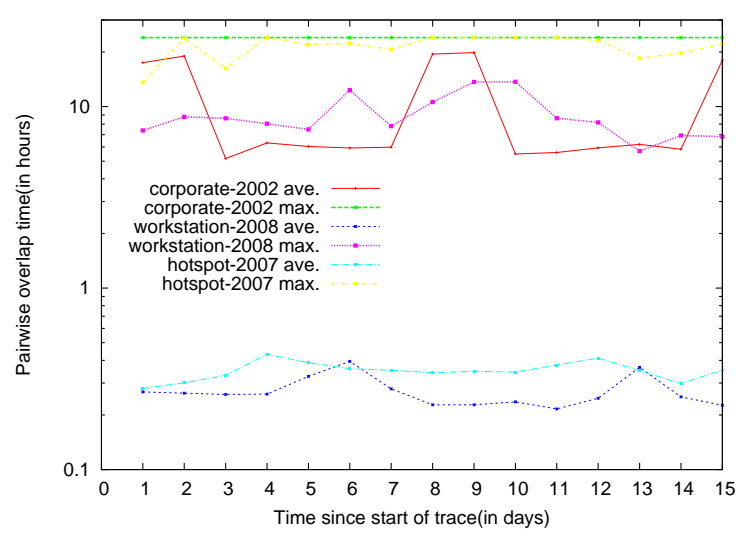

(b) Overlap time among user pairs

Figure 11. Availability parameters that affect the lag metrics

The number of node pairs is $O\left(n^{2}\right)$ of the group size, the lag metrics depend on the group size $(O(n))$. The lag metrics depend on the number of updates created; hence given our assumptions, a locale where users are available for long durations will create large numbers of updates which can potentially lead to higher lagAmounts. We plot the average number of updates created per day of our traces in Fig. 11(a). Corporate-2002 users were available for long durations. Hence they were assumed to be creating large number of updates. Users were online for over 0.4 and 0.9 days on weekdays and weekends, respectively. However, the number of users was smaller during the weekends. These values were smaller for the other traces, about 0.02 days for the hotspot-2007 trace and 0.04 days for the workstation-2008 traces.

For distributed scenarios, the lag metrics also depend on the periods when nodes overlap. We plot the amount of pair-wise node overlap for the various traces in Fig. 11(b). The average overlap between pairs of nodes was 0.35 hours for both the hotspot-2007 and workstation2008 traces. However, the duration was about six hours during the weekdays and over 11 hours over the weekend for the corporate-2002 users. Unlike the corporate-2002 trace where some nodes overlapped for 24 hours, the pairs of university users never overlapped for more than 10.5 hours. Thus, the number of updates as well as the rate of update propagation are likely to be high in the corporate-2002 scenario.

We randomly created groups of three, fifteen and thirty users and investigate the effects of the update frequency using the lag and gossip metrics. Three is the smallest meaningful group size for asynchronous propagation while thirty is a large group. We repeat the simulations for fifty different groups.

\subsection{Limitations of asynchronous propagation}

First we analyzed the performance limit. The best performance is achieved in a Svr-ServInit scenario where the update duration is zero seconds; i.e., the server constantly polls all nodes; any updates are immediately pulled by the server and sent to all the other nodes that are also online. Still, updates cannot be sent to nodes that are not online. Thus, the best performance is dependent on the user availability. Also, this policy is not practical because it will incur tremendous network overhead for constantly pulling updates. Practical systems delay this operation and propagate updates at less frequent intervals; we investigate these intervals in Section 4.4.

We plot the lagAmount for groups of size three, fifteen and fifty in Fig. 12. We note that the lag metrics continue to rise throughout the trace duration. This phenomenon is caused by node churn. When a node leaves the system, we continue to compute its lag values for updates created by other nodes. Our propagation policies described in Section 4.4 counteract this effect by reporting the relative difference from the best policy.

The lag amounts were high and depend on the group size. From Figs. 12(a), 12(b) and 12(c), we note that the number of updates that are yet to propagate to a node is as high as forty four days by the end of the trace for the corporate-2002 trace. The session durations for the hotspot-2007 and workstation-2008 traces were smaller (Section 2), producing smaller lag amounts.

Each day, nodes did not receive updates that were created in about $\frac{1}{5}$ days from other nodes. The number of updates accumulated depends on the node session duration and churn; corporate-2002 users exhibit large amounts of pending updates. Unless efforts to improve the wireless user availability are successful, asynchronous mechanisms are not suitable for applications which require tighter propagation.

\subsection{Performance of propagation policies}

Next, we investigate the extra overhead imposed by various update propagation policies as compared to the best policy (Section 4.3). For example, if the lagAmount 


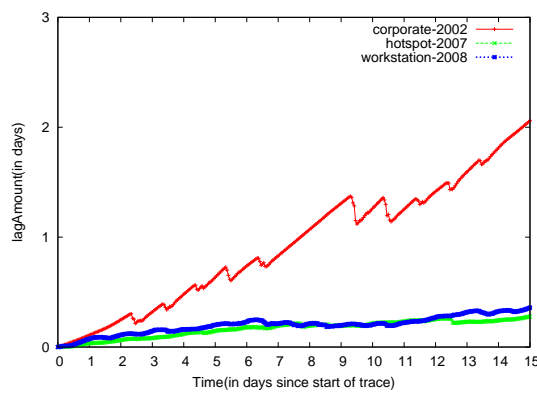

(a) lagAmount, group size $=3$

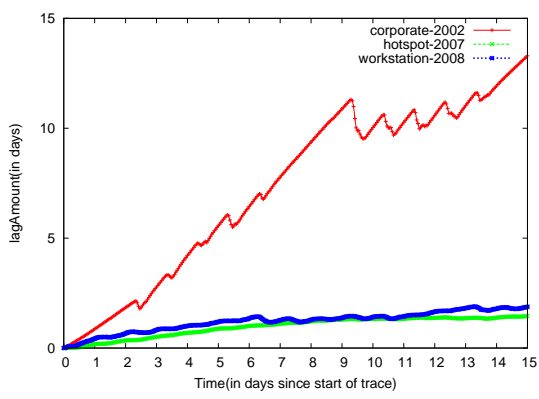

(b) lagAmount, group size $=15$

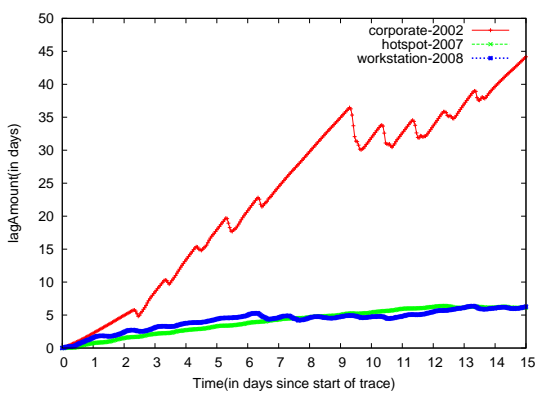

(c) lagAmount, group size $=50$

Figure 12. Best performance achievable for the various traces

on a particular day for the P2P-pull policy was four while the corresponding values for the best policy was three, we report a relative lagAmount of one day. Analyzing the relative costs has the added benefit of eliminating the lag values accumulated due to not propagating updates to nodes that have left the system. However, updates which were created on the node that will leave the system and that was not propagated to other nodes will still affect the relative costs. We prefer a policy that minimizes the relative performance.

Investigation of propagation frequency. Nodes propagate updates when they become online with subsequent propagations at regular intervals. This frequency is application driven and depends on the user availability. If the node becomes unavailable before update propagation, then the updates created during this session will be delayed. It is impractical to require nodes to propagate updates before going offline. Hence, we investigate whether we can predict when a node will go offline and correspondingly adapt the update frequency. We investigate history based approaches that use the past session durations to predict the expected session duration. For this analysis, we use various history values of one, two and three. For each of these values, we choose base frequencies of five min., fifteen min., thirty min. or an hour. For each base frequency, if the predicted session duration was less than the predicted duration, we reduce the update frequency to the predicted value. We tabulate the cumulative percentage of times when a node goes offline before subsequent update propagation for our traces in Table 1. The table is read as follows: for the workstation2008 trace, using a base frequency of an hour, 90.1\% of the time, the node will go offline before subsequent propagation. Given the average session duration of 20 mins. (Section 2), most of these updates are unduly delayed until the node came back online; average time between sessions was 1.78 hours for this trace (Section 2 ). The lag values are reduced when the times when no further updates were propagated was low; using the last session duration can reduce this value to $48.9 \%$.

From Table 1, we note that the percentage of time when nodes did not subsequently propagate updates was higher for workstation-2008. In all the traces, the best performance was achieved when the base update frequency was high; this observation needs to be reconciled with the number of unnecessary gossips. An adaptive policy that used the recent past session duration was also effective. We investigate our propagation policies using the adaptive as well as application specified propagation durations.

P2P-pull. We plot the relative lagAmount and the number of gossips and unnecessary gossips in Fig. 13. As nodes come online, they pull updates from other group members that are also online at frequent intervals that are either fixed or adaptive (Section 4.4). The times that nodes come online are unpredictable. Hence the pull operations to propagate updates created at any node to other nodes is also random. Note that the adaptive duration allows the local node to pull updates from other nodes before going offline; it does not affect when the local updates are sent to other nodes. Adaptive policies only made significant improvements in the workstation-2008 scenario.

Consider the relative lagAmount (Fig. 13(a)). We note that the lagAmounts continue to increase because of the residual updates left in a node that were not pulled by other nodes before it left the system; the best policy (Section 4.3) would have propagated these updates. This effect was pronounced in the hotspot-2007 scenarios. For a group of size 15 in hotspot-2007 scenario with an adaptive policy, base frequency of one hour and history depth of one, the relative lagAmount on the fifteenth day was over 1.2 days; i.e., each pair of nodes did not have about $\frac{1.2}{14}=0.086$ days worth of updates. These values were slightly bigger for smaller groups which had fewer opportunities for update propagation (pairwise lagAmount of $\frac{0.2}{2}=0.1$ ). We also observed that the adaptive policy for the workstation-2008 scenario 


\begin{tabular}{|c|c|c|c|c|c|c|c|c|c|c|c|c|c|c|c|c|}
\hline \multirow{2}{*}{ trace } & \multicolumn{4}{|c|}{ base freq $=5 \mathrm{~min}$} & \multicolumn{4}{|c|}{ base freq $=15 \mathrm{~min}$} & \multicolumn{4}{|c|}{ base freq $=30 \mathrm{~min}$} & \multicolumn{4}{|c|}{ base freq $=60 \mathrm{~min}$} \\
\hline & 0 & 1 & 2 & 3 & 0 & 1 & 2 & 3 & 0 & 1 & 2 & 3 & 0 & 1 & 2 & 3 \\
\hline corporate- 2002 & 1.4 & 1.3 & 1.4 & 1.4 & 10.0 & 8.9 & 9.7 & $\overline{99.7}$ & 17.5 & 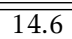 & 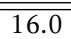 & 10.3 & 28.5 & 22.3 & 24.7 & 25.2 \\
\hline hotspot-2007 & 2.5 & 2.4 & 2.5 & 2.5 & 20.1 & 16.7 & 17.4 & 17.2 & 38.8 & 28.7 & 30.2 & 30.1 & 60.6 & 39.4 & 41.4 & 41.1 \\
\hline workstation-2008 & 18.9 & 15.7 & 17.3 & 17.8 & 40.0 & 30.1 & 33.9 & 35.5 & 63.4 & 41.6 & 47.1 & 49.2 & 90.1 & 48.9 & 52.4 & 53.7 \\
\hline
\end{tabular}

Table 1. Cumulative percentage of times when node went offline before subsequent update propagation

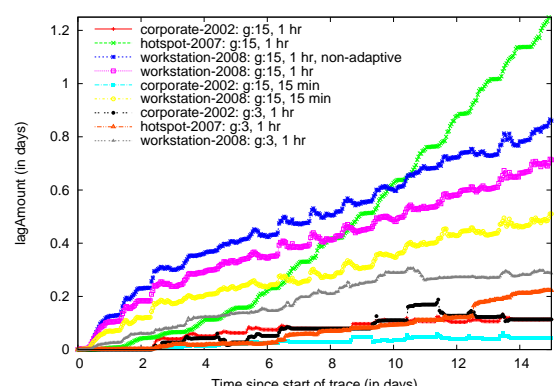

(a) lagAmount

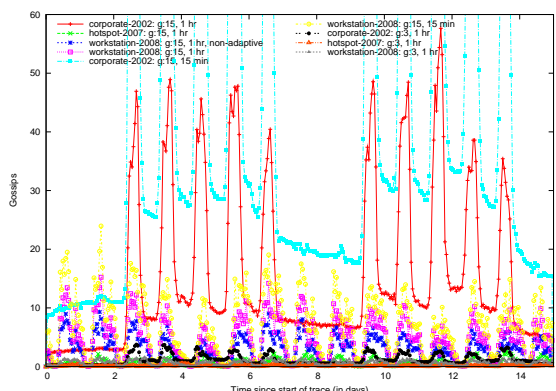

(b) numGossips

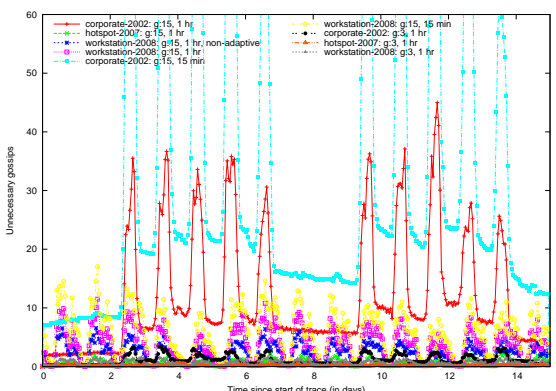

(c) numWGossips

Figure 13. P2P-pull (adaptive duration by default)

showed a residual relative lagAmount of 0.7 days vs 0.88 days for a non-adaptive policy.

These updates were spread over long durations, especially in the hotspot-2007 scenario. The improvements of the adaptive policy was more modest even for the workstation-2008 setting. Investigating the number of gossips and the number of unnecessary gossips in Figs. 13(b) and 13(c), respectively, we note the high number of gossips as well as unnecessary gossips in the corporate setting. For other scenarios which exhibit poorer user availability, the number of gossips were low; about ten for groups of size fifteen. Later, we show that these parameters are competitive with server mediated approaches.

P2P-push. For the P2P-push policy (Fig. 14), the system is far more sensitive to the propagation rate of the local node. Note that for P2P-pull, it is the responsibility of other nodes to pull local updates. For a group of size 15 , there are 14 other nodes which are attempting to perform this propagation vs one for the $P 2 P$-push policy. An adaptive policy may push its updates to another node before going offline. However, any local update that was not pushed by the source to another node will not propagate to any other nodes; increasing the overall lag metrics. Overall, the system performance is slightly worse than the performance of the P2P-pull policy. An adaptive policy for workstation-2008 setting for a group of size 15 with base propagation frequency of one hour experiences an lagAmount of 0.75 days (as compared to 0.7 days for the $P 2 P$-pull policy). Hence, $P 2 P$-pull is preferable to P2P-push.

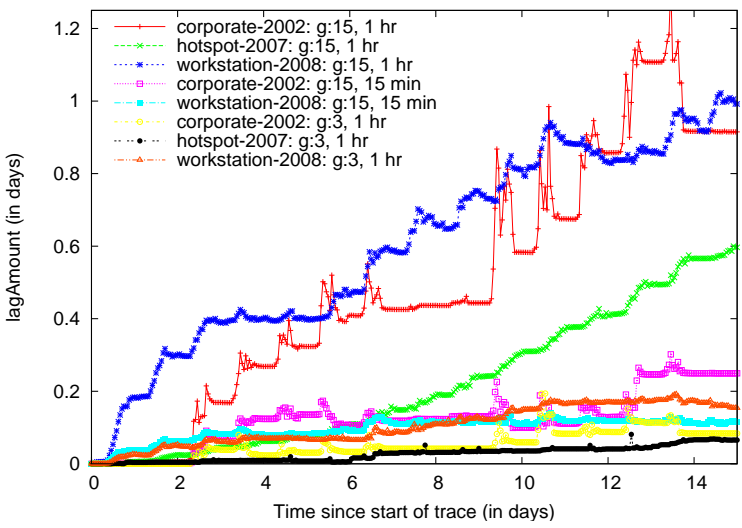

(a) lagAmount

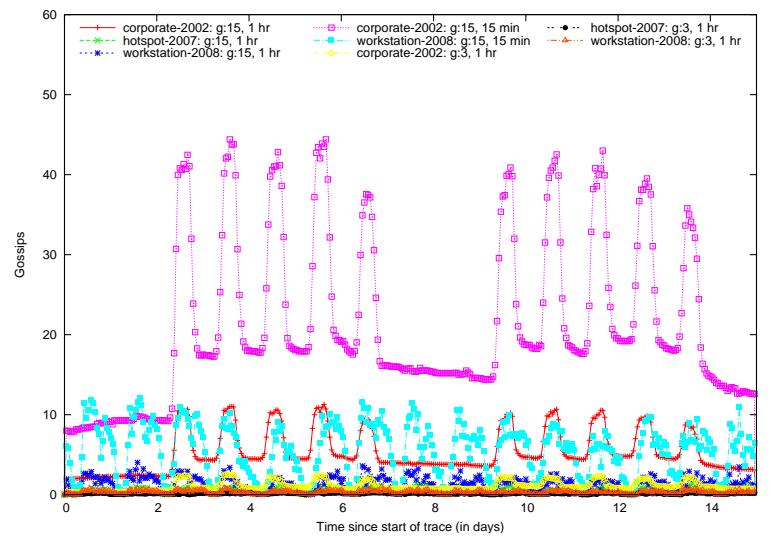

(b) numGossips

Figure 15. Svr-Servlnit 


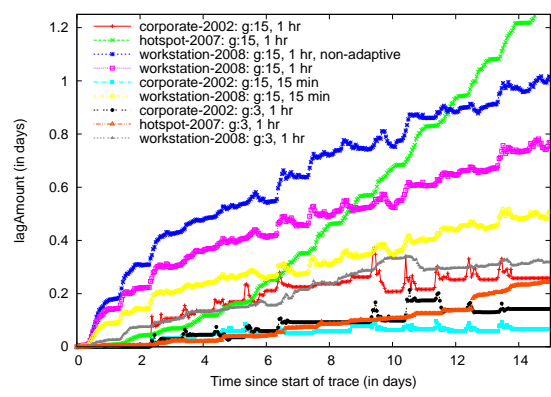

(a) lagAmount

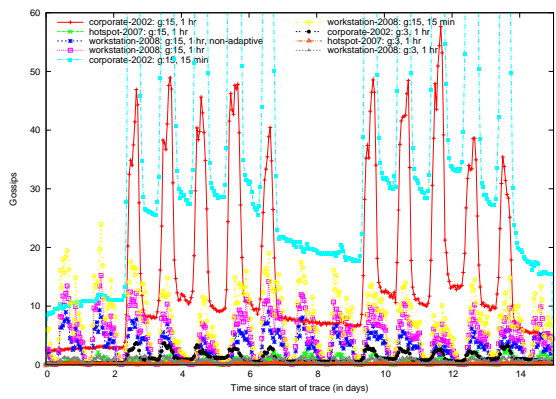

(b) numGossips

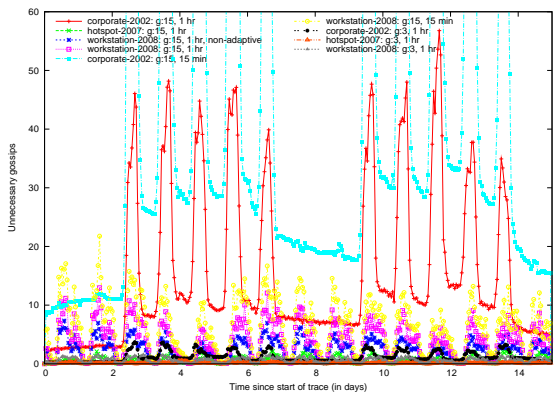

(c) numWGossips

Figure 14. P2P-push (adaptive duration by default)

Svr-Servlnit. Next, we investigate the relative performance of server assisted approaches. The server periodically pulls new updates from online nodes and sends them to other online nodes. We plot the relative lagAmount and the number of gossips in Fig. 15. Note that the server cannot adapt to the session durations of individual nodes; if a particular node came online and went offline between the propagation interval, then its updates are not propagated to other nodes even though the server is continuously available. Each gossip as compromised of two operations; a pull of contents form a particular node and push these contents to other nodes. All the gossips in this scenario are useful.

From Fig. 15(a), we observed that the lagAmount progressively increases with time. Even if the server was continuously available, it is possible for a node to come online and go offline without propagating its updates to other nodes (especially if the session duration was below the propagation rate). For small group sizes, the Svr-ServInit policy exhibits better performance than distributed approaches. For example, for groups of size three in a workstation-2008 with base updates every hour, the adaptive P2P-pull policy experienced a relative lagAmount of 0.3 days at the end of the $15^{\text {th }}$ day. However, the Svr-ServInit policy for similar settings experienced a lagAmount of 0.15 days. However, the distributed approaches are more competitive for larger groups. For the Svr-ServInit policy in the workstation2008 scenario with a group size of 15 and a propagation frequency of an hour, the relative lagAmount is about a day. The corresponding values for an adaptive $P 2 P$ pull was only 0.7 days. The improvements were even more pronounced for groups of size 50. Since the distributed approaches allow all the nodes to initiate the propagation operation, $\mathrm{P} 2 \mathrm{P}$ schemes achieve better randomization of update propagation.

Svr-Nodelnit. Svr-NodeInit policy (Fig. 16) is similar to the P2P-push policy in that nodes periodically sends its updates to the server while simultaneously downloading new updates from other nodes. Thus, this

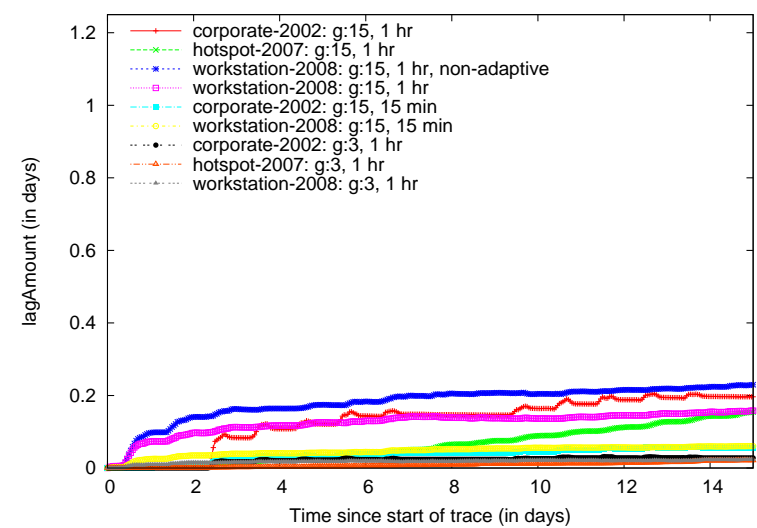

(a) lagAmount

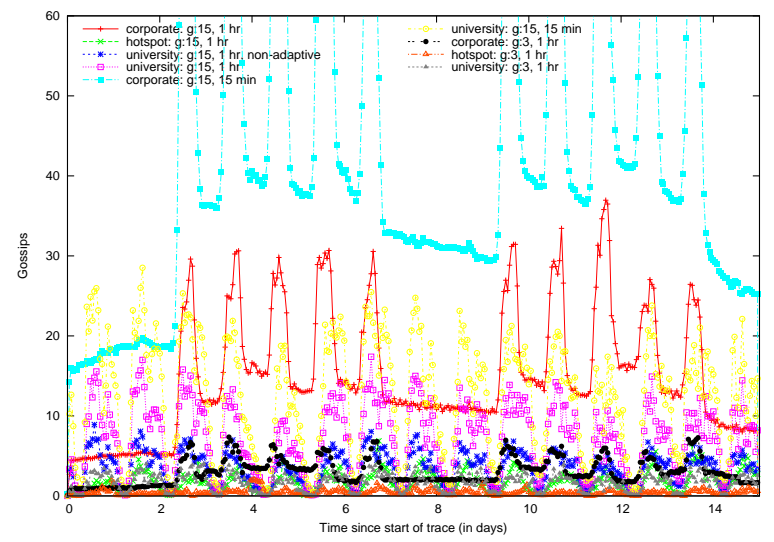

(b) numGossips

Figure 16. Svr-Nodelnit

policy leverages the availability of server nodes and propagates its updates quickly. Fig. 16(a) shows that even after fifteen days, the lagAmount was a modest 0.2 days (this value can be as high as 1.2 days for the other propagation mechanisms).

Summary. We investigated the performance bounds of a best policy that instantaneously propagated the updates created at any node to other nodes. We then compared 
the performance of several distributed and server mediated approaches with this policy. The fundamental limitation of these practical policies was the mismatch between the propagation rates and the times when nodes go offline before propagating their updates to other nodes. Frequent propagations correspondingly increase the number of unnecessary gossips; an adaptive policy that tuned the frequencies to the node session duration improved the system performance for distributed approaches. These adaptive policies only required the last session duration of any node. We showed that the Svr-NodeInit policy achieved the best performance with the P2P-pull policy exhibiting competitive performance. Overall, distributed policies are competitive, especially for larger groups.

\subsection{Peer selection mechanisms}

Increasing the propagation rate improves system performance while also requiring a higher number of gossips. One approach to address this concern is to choose higher propagation rates while proportionately reducing the number of nodes to propagate updates. We investigate this tradeoff for our wireless users.

We plot the relative performance for varying the number of peers used for update propagation using the non-adaptive P2P-pull policy for groups of size 15 in Fig. 17. We reduce the percentage of users from $100 \%$ to $50 \%$ and $25 \%$ while increasing the propagation rates from 1 hour to $30 \mathrm{~min}$. and $15 \mathrm{~min}$. For the corporate-2002 trace, choosing fewer nodes has relatively minor effect on the lagAmounts. At the end of fifteen days, the lagAmount was about 0.1 days for propagating to $100 \%$ as well as $50 \%$ of the nodes (while doubling the propagation rates). Propagating to $25 \%$ of the nodes (while quadrupling the propagation rate) increased the lagAmount to about 0.15 days. The corresponding reduction in unnecessary gossips was from 24 to 16 and 14, respectively. Reducing the percentage of nodes to propagate updates while increasing the update frequency is a viable approach for large corporate-2002 groups. For the hotspot-2007 trace, reducing the frequency increased the lagAmount from 1.3 days to 1.5 days with small differences in the number of unnecessary gossips. On the other hand, for the workstation-2008 trace, reducing the percentage of online nodes to propagate updates from $100 \%$ to $50 \%$ and $25 \%$ (while exponentially increasing the propagation rates) drastically worsened the lagAmount from about 0.8 days to 1.3 days and 1.6 days, respectively. The number of unnecessary gossips showed a small improvement.

A similar analysis for groups of size fifty (Fig. 18) showed a worse performance for both the hotspot-2007 and the workstation-2008 traces. For the hotspot-2007 traces, the lagAmounts increased from 2 days to 2.6 and 3.2 days, respectively. For the workstation-2008 traces, the lagAmounts increased from 0.6 days to 1.2 and 1.55 days, respectively. The number of unnecessary gossips also worsened for the corporate-2002 trace; from 50 gossips to 225 and 125 gossips, respectively. For scenarios which exhibit poorer availability, reducing the percentage of nodes while increasing the update propagation rate is not a viable option.

\section{Related work}

Bolosky et al. [5] analyzed the availability of wired corporate desktops. They [32] showed that the availability was sufficient to support up to four nines availability using three replicas in the Farsite [30] storage system.

Prior studies analyzed user mobility, the network traffic and load characteristics of wireless users in various deployment scenarios. We used some of these traces to analyze the behavior of group communications amongst these wireless users. Tang et al. [40] monitored the WLAN network in a university building using tcpdump traces, AP SNMP and authentication logs in order to understand the network traffic and load characteristics. Analyzing the authentication logs, they observed that session lengths were longer than twelve hours. Kotz et al. [16] investigated a campus wide WLAN network using packet dumps (tcpdump) as well as access point SNMP and association logs. They note that HTTP protocol and file backups dominated the wireless traffic. They also conducted a followup analysis [3] on the evolution of their campus WLAN deployment which showed that the traffic had evolved to include significant amounts of traffic for streaming multimedia, VOIP and P2P traffic. They also observed that on-campus traffic outstripped offcampus traffic, an indication of the importance of group communications among wireless users. Chandra et al. [9] analyzed the popularity and nature of objects shared by iTunes users in a campus setting. Balachandran et al. [22] analyzed the wireless traffic from SIGCOMM 2001 participants and showed that Web and SSH dominated the traffic at over $64 \%$ of the traffic. Balazinska et al. [20] analyzed the access point load and user mobility behavior of corporate wireless LAN users by using SNMP probes of the access points. Papadopouli et al. [41] analyzed the wireless user mobility pattern at the UNC campus. On the Verizon hotspot network, Blinn et al. [26] observed that $45.74 \%$ of the user sessions lasted more than one hour. Hsu et al. [42] presented a comprehensive analysis of the user mobility behavior across four different university campuses using access point logs. McNett et al. [43] focused their mobility analysis to PDA users in a university setting.

Song et al. [44] used the AP records to synthesize contact patterns among wireless users. They note that asynchronous update propagation can be unacceptably 


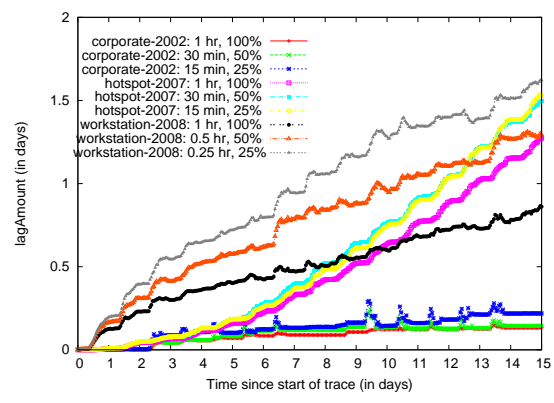

(a) lagAmount

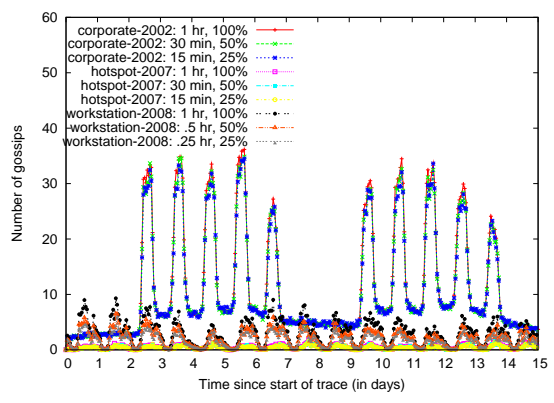

(b) numGossips

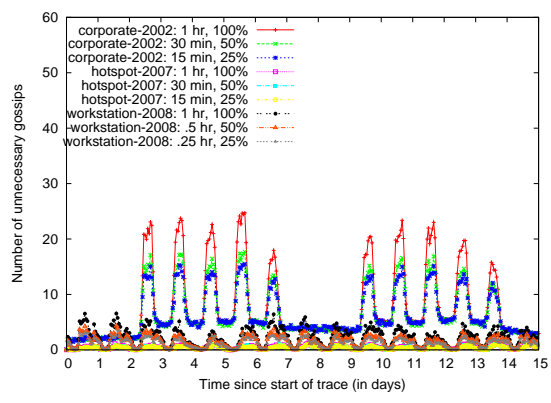

(c) numWGossips

Figure 17. Tradeoff between update frequency and choosing fewer online nodes (group size: 15, $P 2 P$-pull)

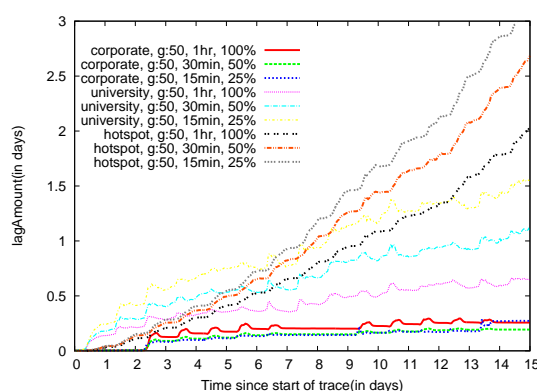

(a) lagAmount

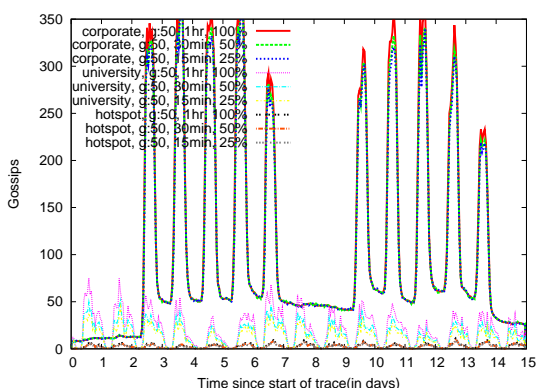

(b) numGossips

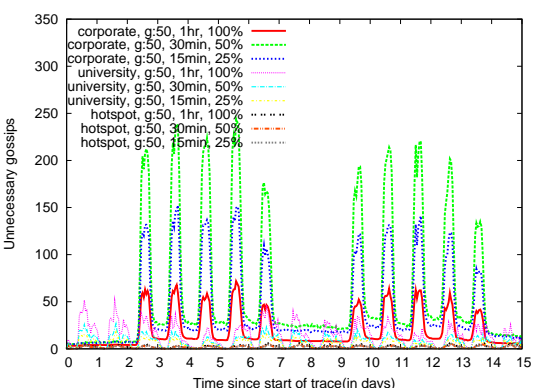

(c) numWGossips

Figure 18. Tradeoff between update frequency and choosing fewer online nodes (group size: 50, P2P-pull)

long, especially among casual users (some users never again meet each other). We assume the availability of a wireless distribution infrastructure. This allowed us to ignore the spatial mobility patterns of the users; we consider any two nodes that were online anywhere on the wireless trace to be accessible. We expect our node availability to be far better than that was observed using user vicinity contact measurements. However, we observed limited system performance. This places serious doubts on the viability of mechanisms that require collaborating groups be co-located ([44]).

Demers et al. [37] describe epidemic algorithms and the parameters that affect system performance. Epidemic algorithms are designed to asynchronously propagate updates; particularly in scenarios with poor user availability. They operate without strict bounds on the time to propagate updates to all the participants. Birman et al. [38] surveyed the recent developments on the strengths and limitations of gossip protocols. They showed that gossip based protocols are not designed to expeditiously propagate updates. We use empirical wireless user availability data to investigate the impact of the propagation parameters.

Vahdat et al. [39] used epidemic routing to propagate updates in an ad hoc network. They simulated a random node mobility and showed that epidemic routing achieved eventual delivery of $100 \%$ of messages. Similarly, Davis et al. [45] investigated propagation among wearable computers using simulated human mobility. They investigated the effects of message duplication and buffer overhead. Recently, delay tolerant network technologies (DTN) are used to asynchronously propagate updates among a set of clients. Fall [46] introduced a network architecture that operated without continuous network connectivity among the participating nodes. In their followup work, Jain et al. [47] investigated the routing behavior across a DTN. They used simulations and progressively increased the amounts of network topology information available to the routing mechanism. They showed that the systems performed better with the addition of more topology information. We validate propagation rates using empirical node availability.

Bakhshi et al. [48] surveyed analysis techniques for gossiping protocols. Our evaluation metrics were influenced by consistency count metric (Kuenning et al. [49]). Jelasity et al. [50] addressed the problem of selecting peers for gossiping. Kwiatkowska et al. [51] evaluated gossip protocols using probabilistic model checking. In contrast to simulation based studies, they provide both an exhaustive search of all possible 
behaviors of the system, including best and worstcase scenarios and exact quantitative results. They were concerned with identifying the set of gossip peers; each node maintains a relatively small local membership table providing a partial view of the network. Using empirical wireless users availability, we show that the system should gossip with all the available peers.

Saito et al. [52] surveyed a number of optimistic replication algorithms. Bayou [53] provides an user-level storage for asynchronous collaborative applications. Nodes exchange version vectors to identify updates that needed to be exchanged during a pair-wise anti-entropy protocol. Updates eventually reach all the participants. The system provides some bounds by using a primary commit protocol. Refdbms [54] used a similar antientropy protocol to disseminate bibliographic entries. Allavena et al. [55] described a scalable gossip-based for local view maintenance. Khelil et. al. [56] developed an epidemic model for an information diffusion algorithm. Motani et. al. [57] developed a wireless virtual social network (PeopleNet) which mimics the way people seek information via social networking. It used the infrastructure to propagate queries of a given type to users in specific geographic locations, called bazaars. Within each bazaar, the query was further propagated between neighboring nodes via a peer-to-peer connectivity until a query match. Rivière et al. [58] described an architecture to develop and reuse epidemic based systems. Zhuang et al. [59] described an Internet indirection infrastructure [60] among mobile users. Our empirical analysis of the availability pattern of wireless users showed that the system that exclusively used other peers was likely to enjoy similar performance to a system that used servers for assistance in propagation, especially for larger group sizes.

\section{Conclusions}

Modern wireless devices are resource rich and ubiquitous. In this work, we analyzed the behavior of various group communications mechanisms at a university, corporate lab, conference venue and a hotspot federation using empirical user availability traces. Our analysis is agnostic to the requirements of specific applications, both in terms of the frequency of updates to the shared contents as well as the size of these updates. We show that the availability behavior was better in corporate settings where the users were available for longer durations with a larger percentage of the wireless users available on weekdays. The session durations were smaller in academia and are becoming shorter. The systems exhibited constant node churn which places heavy load on asynchronous group communication systems that need to transmit prior updates to these newer nodes. We developed a lag metric to measure the performance of our system. We show that, regardless of the propagation policy, factors such as node availability and node churn play an important role in the system performance. Distributed policies are competitive, especially for larger groups. We show that for wireless users, propagation mechanisms should propagate update to all the nodes. Our work highlights the need for robust expiration mechanisms for older updates. The amount of updates created is small enough to make distributed approaches viable. We built a middleware called Yenta to implement the lessons learn from our analysis. The algorithms also form the basis for our flockfs moderated collaboration group-ware. Stable versions of Yenta will be published at http://yenta. sourceforge. net/.

Acknowledgements. We thank Kevin Smyth for his help in configuring the APs. This work was supported in part by the U.S. National Science Foundation (CNS-0447671).

\section{References}

[1] Della Cava, M.R. (2006), Working out of a 'third place', USA Today. 10/5/2006.

[2] iSuppli Corp. (2008), Notebook pc shipments exceed desktops for first time in Q3, i suppli . com/NewsDetail. aspx? ID $=19823$.

[3] Henderson, T., Kotz, D. and Abyzov, I. (2004) The changing usage of a mature campus-wide wireless network. In MobiCom '04: 187-201.

[4] BuddeCoмm (2008), 2008 global wireless broadband next generation mobility.

[5] Bolosky, W.J., Douceur, J.R., Ely, D. and Theimer, M. (2000) Feasibility of a serverless distributed file system deployed on an existing set of desktop pcs. In $A C M$ SIGMETRICS: $34-43$.

[6] Rosenwald, M.S. (2009) Digital nomads choose their tribes. Washington Post .

[7] Kotz, D. and Henderson, T. (2005) Crawdad: A community resource for archiving wireless data at dartmouth. IEEE Pervasive Computing 4(4): 12-14.

[8] Chen, G. and Kotz, D. (2005) Structural Analysis of Social Networks with Wireless Users. Tech. Rep. TR2005-549, Dept. of Computer Science, Dartmouth College.

[9] Chandra, S. and Yu, X. (2007) Share with thy neighbors. In ACM/SPIE Multimedia Computing and Networking (MMCN 2007) (San Jose, CA).

[10] (2008), The new oases, The Economist.

[11] Chandra, S. and Regola, N. (2009) flockfs, a moderated group authoring system for wireless workgroups. In Mobiquitous '09 (Toronto, Canada).

[12] Chandra, S. (2011) Moderated group authoring system for campus-wide workgroups. IEEE Transactions on Mobile Computing 99(PrePrints).

[13] Kotz, D., Henderson, T. and Abyzov, I. (2004), CRAWDAD trace set dartmouth/campus/snmp (v. 200411-09).

[14] Henderson, T. and Kotz, D. (2007), CRAWDAD trace dartmouth/campus/syslog/05_06 (v. 2007-02-08), http://crawdad.cs.dartmouth.edu/dartmouth/campus/ syslog/05_06. 
[15] Henderson, T. (2006), CRAWDAD tool tools/process/syslog/syslog_parser (v. 2006-11-01), http://crawdad.cs.dartmouth.edu/tools/process/syslog /syslog_parser.

[16] Kotz, D. and Essien, K. (2002) Analysis of a campuswide wireless network. In ACM MobiCom '02: 107-118.

[17] Zero configuration networking (zeroconf), http://www. zeroconf.org/.

[18] Voida, A., Grinter, R.E., Ducheneaut, N., Edwards, W.K. and Newman, M.W. Listening in: practices surrounding itunes music sharing. In CHI '05: 191-200.

[19] Balazinska, M. and Castro, P. (2003), CRAWDAD data set ibm/watson (v. 2003-02-19), http://crawdad.cs. dartmouth. edu/ibm/watson.

[20] Balazinska, M. and Castro, P. (2003) Characterizing mobility and network usage in a corporate wireless localarea network. In MobiSys '03: 303-316.

[21] Balachandran, A., Voelker, G.M., Bahl, P. and RANGAN, P.V. (2002), CRAWDAD data set ucsd/sigcomm2001 (v. 2002-04-23), http://crawdad.cs.dartmouth.edu/ucsd/sigcomm2001.

[22] Balachandran, A., Voelker, G.M., Bahl, P. and RANGAN, P.V. (2002) Characterizing user behavior and network performance in a public wireless lan. In ACM SIGMETRICS '02: 195-205.

[23] Schulman, A., Levin, D. and Spring, N. (2009), CRAWDAD data set umd/sigcomm2008 (v. 2009-03-02), Downloaded from http://crawdad.cs.dartmouth.edu/umd/sigcomm 2008.

[24] Lenczner, M., Gregoire, B. and Proulx, F. (2007) CRAWDAD trace ilesansfil/wifidog/session/04_07 (v. 2007-08-27), http://crawdad.cs.dartmouth.edu/ ilesansfil.

[25] Chinchilla, F., Lindsey, M. and Papadopouli, M. (2004) Analysis of wireless information locality and association patterns in a campus. In IEEE INFOCOM '04.

[26] Blinn, D.P., Henderson, T. and Kotz, D. (2005) Analysis of a Wi-Fi hotspot network. In International Workshop on Wireless Traffic Measurements and Modeling (WiTMeMo '05): 1-6. URL http: //www.cs. dartmouth . edu/ dfk/papers/blinn: hotspot.pdf.

[27] Ellis, C.A. and GibBs, S.J. (1989) Concurrency control in groupware systems. SIGMOD Rec. 18(2): 399-407.

[28] Morris, J.H., Satyanarayanan, M., Conner, M.H. Howard, J.H., Rosenthal, D.S. and Smith, F.D. (1986) Andrew: a distributed personal computing environment. Commun. ACM 29(3): 184-201.

[29] Shepler, S., Callaghan, B., Robinson, D., Thurlow, R. Beame, C., Eisler, M. and Noveck, D. (2003), Network file system (nfs) version 4 protocol, RFC 3530.

[30] Adya, A., Bolosky, W.J., Castro, M., Cermak, G., Chaiken, R., Douceur, J.R., Howell, J. et al. (2002) Farsite: federated, available, and reliable storage for an incompletely trusted environment. SIGOPS Oper. Syst. Rev. 36(SI): 1-14.

[31] Vazhkudai, S.S., Ma, X., Freeh, V.W., Strickland, J.W., Tammineedi, N., Simon, T. and Scott, S.L. (2006) Constructing collaborative desktop storage caches for large scientific datasets. Trans. Storage 2(3): 221-254.

[32] Douceur, J.R. and Wattenhofer, R.P. (2001) Optimizing file availability in a secure serverless distributed file system. In 20th IEEE Symposium on Reliable Distributed Systems (New Orleans, LA): 4-13.

[33] Yu, X. and Chandra, S. (2008) Campus-wide asynchronous lecture distribution using wireless laptops. In ACM/SPIE MMCN'08 (San Jose, CA), 6818.

[34] Satyanarayanan, M., Kistler, J.J., Kumar, P., Okasaki, M.E., Siegel, E.H. and Steere, D.C. (1990) Coda: A highly available file system for a distributed workstation environment. IEEE Transactions on Computers 39(4).

[35] Page, T.W., Guy, R.G., Heidemann, J.S., Ratner, D., Reiher, P., Goel, A., Kuenning, G.H. et al. (1998) Perspectives on optimistically replicated peerto-peer filing. Software-Practice and Experience 28(2): 155-180. URL http://www.isi.edu/ johnh/PAPERS/ Page98a.html.

[36] Demers, A., Petersen, K., Spreitzer, M.J., Terry, D., Theimer, M. and Welch, B. (1994) The bayou architecture: support for data sharing among mobile users. In Workshop on Mobile Computing Systems and Applications (Santa Cruz, CA): 2-7.

[37] Demers, A., Greene, D., Hauser, C., Irish, W., Larson, J., Shenker, S., Sturgis, H. et al. (1987) Epidemic algorithms for replicated database maintenance. In PODC: $1-12$

[38] Birman, K. (2007) The promise, and limitations, of gossip protocols. SIGOPS Oper. Syst. Rev. 41(5): 8-13.

[39] Vahdat, A. and Becker, D. (2000) Epidemic Routing for Partially Connected Ad Hoc Networks. Tech. Rep. CS-200006, Duke University.

[40] TANG, D. and BAKER, M. (2000) Analysis of a local-area wireless network. In ACM Mobicom '00: 1-10.

[41] Papadopouli, M., Shen, H. and Spanakis, M. (2005) Characterizing the duration and association patterns of wireless access in a campus. In 11th European Wireless Conference (Nicosia, Cyprus).

[42] Jen Hsu, W. and Helmy, A. (2005) IMPACT: Investigation of Mobile-user Patterns Across University Campuses using Wlan Trace Analysis. Tech. rep., USC.

[43] McNett, M. and Voelker, G.M. (2005) Access and mobility of wireless pda users. SIGMOBILE Mob. Comput. Commun. Rev. 9(2): 40-55.

[44] Song, L. and Koтz, D. (2007) Evaluating opportunistic routing protocols with large realistic contact traces. In ACM MobiCom workshop on Challenged Networks (CHANTS 2007).

[45] Davis, J.A., FAgG, A.H. and Levine, B.N. (2001) Wearable computers as packet transport mechanisms in highlypartitioned ad-hoc networks. In IEEE Intl. Symp. on Wearable Computers: 141-148.

[46] FALL, K. (2003) A delay-tolerant network architecture for challenged internets. In SIGCOMM '03: 27-34.

[47] Jain, S., Fall, K. and Patra, R. (2004) Routing in a delay tolerant network. In Sigcomm '04: 145-158.

[48] Bakhshi, R., Bonnet, F., Fokkink, W. and Haverkort, B. (2007) Formal analysis techniques for gossiping protocols. SIGOPS Oper. Syst. Rev. 41(5): 28-36.

[49] Kuenning, G.H., Bagrodia, R., Guy, R.G., Popek, G.J., ReIHER, P.L. and WANG, A.I. (1998) Measuring the quality of service of optimistic replication. In ECOOP '98: Workshop ion on Object-Oriented Technology (London, UK: Springer-Verlag): 319-320. 
[50] Jelasity, M., Guerraoui, R., Kermarrec, A.M. and van Steen, M. (2004) The peer sampling service: experimental evaluation of unstructured gossip-based implementations. In Middleware '04 (New York, NY, USA: Springer-Verlag New York, Inc.): 79-98.

[51] Kwiatkowska, M., Norman, G. and Parker, D. (2008) Analysis of a gossip protocol in prism. SIGMETRICS Perform. Eval. Rev. 36(3): 17-22.

[52] Saito, Y. and Shapiro, M. (2005) Optimistic replication. ACM Comput. Surv. 37(1): 42-81.

[53] Terry, D.B., Theimer, M.M., Petersen, K., Demers, A.J., Spreitzer, M.J. and Hauser, C.H. (1995) Managing update conflicts in bayou, a weakly connected replicated storage system. In ACM symposium on Operating systems principles (Copper Mountain, CO): 172-182.

[54] Golding, R.A., Long, D.D.E. and Wilkes, J. (1994) The refdbms distributed bibliographic database system. In Winter Usenix Conference (San Francisco, CA): 47-62.

[55] Allavena, A., Demers, A. and Hopcroft, J.E. (2005) Correctness of a gossip based membership protocol. In
PODC '05 (New York, NY, USA: ACM): 292-301.

[56] Khelil, A., Becker, C., Tian, J. and Rothermel, K. (2002) An epidemic model for information diffusion in manets. In MSWiM '02: 54-60.

[57] Motani, M., Srinivasan, V. and Nuggehalli, P.S. (2005) Peoplenet: engineering a wireless virtual social network. In MobiCom '05: 243-257.

[58] Étienne Rivière, Baldoni, R., Li, H. and Pereira, J. (2007) Compositional gossip: a conceptual architecture for designing gossip-based applications. SIGOPS Oper. Syst. Rev. 41(5): 43-50.

[59] Zhuang, S., Lai, K., Stoica, I., Katz, R. and Shenker, S. (2003) Host mobility using an internet indirection infrastructure. In MobiSys '03 (New York, NY, USA: ACM): 129-144.

[60] Stoica, I., Adkins, D., Zhuang, S., Shenker, S. and SuRANA, S. (2004) Internet indirection infrastructure. IEEE/ACM Trans. Netw. 12(2): 205-218. 